\title{
Die Größe ,Gewicht` im deutsch-italienischen Sprachvergleich
}

\author{
Gudrun Bukies (Cagliari)
}

\begin{abstract}
The topic of this article is 'weight' in the German-Italian language comparison. Which linguistic means are used to refer to weight in German (Gewicht) and what are the Italian equivalents? The material which has been collected is based on monolingual German and Italian dictionaries, reference works and text corpora as well as on bilingual German-Italian dictionaries and text excerpts.

The classification of the so-called weight designations including derivatives, composites and word combinations is carried out from an etymological and lexical perspective. In addition to the dictionary entries, German-Italian translation examples show further equivalents of terms and expressions with regards to 'weight' in this language pair.
\end{abstract}

\section{Untersuchungsgegenstand}

Für kaum eine andere Größe gibt es im Deutschen so zahlreiche Bezeichnungen wie für Gewicht. ${ }^{1}$ Dies hängt möglicherweise damit zusammen, dass das Wiegen und Wägen seit Jahrtausenden ein wichtiger Bestandteil des menschlichen Lebens ist. Wie archäologische Funde belegen, war die Waage bereits in den Hochkulturen bekannt und zählt zu den ,ältesten Erfindungen der Menschheit“ (Haustein 2001: 24). Während man sich bei den frühgeschichtlichen Gewichtsordnungen an objektiv vorhandenen Größen, Naturphänomenen und astronomischen Beobachtungen orientierte, wurden Gewichtsmessungen im Laufe der Zeit immer komplexer und technischer. Heute gehört das Messwesen/die Metrologie stärker denn je zu unserem Alltag und gilt zusammen mit Maßsystemen und Messpraxis als „Abbild [...] der Evolution der menschlichen Gesellschaft“ (ibd.: IX).

Im Handel spielen Gewichte seit jeher eine zentrale Rolle. Gewichtseinheiten waren sowohl Grundlage vieler Währungen als auch Namensgeber von Gewichts- bzw. Münzeinheiten wie die Währungsnamen Baht (thail. ,traditionelle Gewichtseinheit'), Drachme (griech. ,Münz-/ Gewichtseinheit'), Lira (von lat. libra ,Pfund'); Mark (,Gewichts- und Werteinheit') (cf. Kluge/Götze 2011: 602), Peso ${ }^{2}$ (span. ,Gewicht') oder Pound (Sterling) bzw. (britisches) Pfund

\footnotetext{
${ }^{1}$ Mit dem hier im Plural verwendeten Begriff „Gewichtsbezeichnung“ sind Wörter gemeint, in denen Gewicht bzw. wicht als Wortstamm vorkommt sowie im weiteren Sinne alle Ausdrücke, die sich auf das Wortfeld ,Gewicht ${ }^{\star}$ beziehen. Mit „Gewichtsbegriff“ sind dagegen die Vorstellungen von Gewicht gemeint und seine unterschiedlichen Bedeutungen (Siehe Kapitel 2). Zur Definition von „Wortfeld“ siehe Fußnote 3.

${ }^{2}$ Zunächst als spanische Silbermünze im 15. Jh. eingeführt und später als Währungseinheit in Spanien bis zur Einführung des Euro gültig. Der Name nimmt Bezug auf lat. pendere, pensum in der Bedeutung ,abwägen“;
} 
bezeugen (cf. Haustein 2001: 80f.). Im fortschreitenden internationalen Austausch stellten die unterschiedlichen Gewichtsordnungen der Handelspartner wegen der aufwändigen Umrechnungen ein unbequemes Hindernis dar. Aus diesem Grund strebte man seit dem 20. Jahrhundert eine Vereinheitlichung des Messwesens an, was 1960 zur Uniformierung durch ein Internationales Einheitensystem (SI) führte.

Im Deutschen gibt es verschiedene Wörter, mit denen man auf ,Gewicht' als eine gegebene, objektiv nachprüfbare Größe verweist wie das Nomen Gewicht, das sich auch in Derivationen und Komposita findet (cf. gewichtig; Gewicht(s)klasse; Höchstgewicht; Schwergewichtler u. v. a.). Aus seiner Grundbedeutung leitete sich im Laufe der Zeit die Verwendung von Gewicht in Wortverbindungen und Redewendungen ab (cf. (großes) Gewicht haben; [...] viel/wenig/ [...] Gewicht beimessen (beilegen); cf. Schemann (1992: 208f; 293); Gewicht auf etwas legen; ins Gewicht fallen (cf. Duden 2020a: 283; Duden 2020b: 323). Ziel dieses Beitrags ist es, die Vielfalt des Sprachmaterials in Verbindung mit der Wortfamilie ${ }^{3}$ und dem Wortfeld ${ }^{4}$ ,Gewicht' darzustellen und - falls vorhanden - die italienischen Entsprechungen dafür anzugeben. Kontrastive Analysen für das Sprachenpaar Deutsch/Italienisch gibt es zu unterschiedlichen sprachlichen und kommunikativen Aspekten u. a. zur Syntax (Koch 2000), zur gesprochenen Sprache (Selig/Morlicchio/Dittmar 2016) sowie zu translatorischen Fragen (Rocco 2020). Die vorliegende Studie ist ein deutsch-italienischer Sprachvergleich auf lexikalisch-semantischer Ebene im Zusammenhang mit der Größe ,Gewicht', deren Relevanz sich aus den unterschiedlichen Bedeutungen einiger Gewichtsbezeichnungen und ihrer Verwendung in den beiden Sprachen ergibt, die zu Fehlschlüssen führen können.

In diesem Zusammenhang wurden die Bedeutungen der deutschen Bezeichnungen und ihrer italienischen Entsprechungen zunächst kontextlos anhand von Erklärungen in den Wörterbüchern gesammelt. Um die entsprechenden Bedeutungsrelationen in den beiden Sprachen zu ermitteln, wurde anschließend eine kontextgebundene Gegenüberstellung durchgeführt.

In Kapitel 2 wird das Wort Gewicht aus etymologischer und lexikalischer Perspektive erläutert. In Kapitel 3 folgt eine Darstellung deutscher Lexeme (Derivationen, Komposita), Wortverbindungen und Redewendungen mit Gewicht als Bestandteil bzw. Komponente zusammen mit italienischen Entsprechungen. In Kapitel 4 werden kurze deutsche Textausschnitte, in denen Gewicht verwendet wird, italienischen Übersetzungen gegenübergestellt und Kapitel 5 enthält eine Zusammenfassung sowie ein abschließendes Fazit.

\footnotetext{
,zuwiegen“ (cf. Brockhaus Enzyklopädie online, s. v. peso). Das Nomen Pensum hat im Deutschen die Bedeutungen 1) „Arbeit, Aufgabe, die innerhalb einer bestimmten Zeit zu erledigen ist“ und 2) „Lehrstoff [Pädagogik, veralt.]“ (Duden online s. v. Pensum).

${ }^{3}$ Wortfamilie wird hier verstanden als „Gruppe von Wörtern, die etymologisch verwandt sind und deren Kernwort (auch als Stamm-, Wurzelmorphem bezeichnet) noch existiert [...]“ (Römer 2010: 359).

${ }^{4}$ Zum Wortfeld ,Gewicht‘ zählen wir nach Römer (2010: 359) „Gruppen von sinnverwandten Wörtern“ wie z. B. Last, Bürde, Schwere sowie neben Nomina auch Adjektive und Verben, im Sinne einer „Extension der Reichweite des Begriffs bis hin zu ganze Handlungsfelder fokussierenden Szenarios“ (ibd.).
} 


\section{Herkunft, Bedeutung und Gebrauch von Gewicht}

Im Deutschen gibt es eine große Vielfalt sogenannter Gewichtsbezeichnungen. Der folgende Teil erläutert die Herkunft des Wortes Gewicht sowie seinen standard- und fachsprachlichen Gebrauch.

\subsection{Herkunft und Bedeutungen}

Gewicht leitet sich von mhd. gwiht(e) ab und wird im Deutschen seit dem 12. Jahrhundert in der Bedeutung ,Schwere eines Körpers‘ verwendet (cf. Kluge/Götze 2011: 357). Laut Fleischer/Barz (1995: 209) handelt es dabei um eine „kombinatorische Derivation“ bestehend aus Ge-wich-t. Das Verb wiegen und das Nomen Gewicht haben eine gemeinsame Wurzel, ebenso wie die abgeleiteten Nomina Wichte ${ }^{5}$, Wichtigkeit und Wucht $t^{6}$ und das Verb gewichten sowie die Adjektive gewichtig bzw. wichtig7. Im Mittelalter entwickelte sich dazu die Bedeutung ,Wert', ,Wichtigkeit', ,Bedeutung, Bedeutsamkeit', später auch ,Betonung ${ }^{6} .{ }^{8}$ Der Bedeutungszusammenhang, der zwischen Gewicht und Wichtigkeit bzw. (ge-)wichtig besteht, wird verständlich, wenn man über die Herkunft von Gewicht, i. e. Gewicht als Größe und über seine Bedeutung im Sinne von ,Schwere‘ reflektiert. Etwas Wichtiges hat somit eine gewisse Schwere und gilt deshalb als bedeutend oder wertvoll. Je nach Verwendungszusammenhang hat Gewicht im deutschen Sprachgebrauch folgende Bedeutungen:

(1a) <ohne Plural $>$ Schwere eines Körpers, die sich durch Wiegen ermitteln lässt; Last; [Beispiele] ein Gewicht von $45 \mathrm{~kg}$; ein geringes, großes Gewicht [...];

(1b) $<$ Physik $>$ Größe der Kraft, mit der ein Körper auf seine Unterlage drückt oder nach unten zieht;

(2) Körper von bestimmter Schwere, der als Maßeinheit zum Wiegen dient; [Beispiele] [...] mehrere Gewichte auf die Waage legen [...].

Duden online s. v. Gewicht

Gemäß der unter (1a) angegebenen Bedeutungserklärung bezeichnet Gewicht (nur im Singular) die ,Schwere eines Körpers' (cf. auch Kluge/Götze 2011: 357) bzw. dient nach (1b) in der Physik als Begriff, im Sinne von „Größe der Kraft, mit der ein Körper [...] nach unten zieht“‘. Dabei wird die ,Schwere' (oder allgemeinsprachlich: das Gewicht) mithilfe von Messgrößen quantifiziert (z. B. ein Gewicht + von $x \mathrm{~kg}+$ haben; it. pesare $x \mathrm{~kg}$; dem entspricht auf Deutsch $x$ Kilo wiegen). Das Gewicht einer Person wird ebenfalls mit Gewicht oder Körpergewicht im Singular bezeichnet.

\footnotetext{
5 „(15. Jh.), Ableitung von wiegen wie Gewicht. Heute nur noch fachsprachlich, seit dem 20. Jahrhundert auch in der Bedeutung ,spezifisches Gewicht““ (Kluge/Götze 2011: 985).

6 ,[...] [Standardwortschatz] (17. Jh.). Übernommen aus [niederdeutsch] wucht, [...] Nebenform zu Gewicht [...].“ (ibd.: 996).

7 „Adj. [Standardwortschatz] (14. Jh.), [mittelhochdeutsch] wihtec, Aus nördlicheren Sprachausprägungen übernommen. [Mittelniederdeutsch] wichtich $(t)$ ist abgeleitet von wichte ,Gewicht ${ }^{\star}[\ldots]$, also eigentlich ,gewichtig‘. Abstraktum: Wichtigkeit.“ (ibd.: 985; Hervorhebungen i. O.).

$8,[\ldots]$ alle bei der Deklamation gemachten Regeln und Bemerkungen werden auch hier zur Grundlage vorausgesetzt. Insbesondere ist aber der Charakter des rhythmischen Vortrags, daß der Gegenstand mit noch mehr erhöhtem, pathetischem Ausdruck deklamiert sein will. Mit einem gewissen Gewicht soll da jedes Wort ausgesprochen werden.“ (Goethe 1733/1982: 255).
} 
Gewicht (auch Gewichtsstück= „ein Metallkörper, der beim Wiegen mit einer Balkenwaage als Vergleichsmasse aufgebracht wird (Wägestück)“ (Brockhaus Enzyklopädie online s. v. Gewichtsstück) bedeutet nach einer weiteren Erklärung (2) „Körper mit einer bestimmten Schwere" (Duden 2018: 441) und wird in dieser Bedeutung meist im Plural verwendet (,er legte drei Gewichte auf die Waage"; ibd.). Gewichte werden beim Wiegen von Gegenständen eingesetzt und bestehen aus schweren Metallen wie z. B. aus Blei, Edelmetallen, Messing oder Stahl. Im Sport wird auf Gewicht in dieser Bedeutung z. B. beim Gewichtheben, Gewichtheber/ -in; den Gewichtsklassen und im Italienischen auch beim Kugelstoßen verwiesen (it. sollevamento pesi/pesistica; pesista; categoria di peso; lancio m del peso; cf. Giacoma/Kolb 2019: 455; 648) (siehe Kapitel 3.1.1).

Gemäß den Bedeutungserklärungen (1a) und (2) bezieht man sich mit Gewicht auf das Messen bzw. Wiegen von Körpern. Dazu gehören ein Messgerät (die Waage - it. la bilancia) sowie Gewichte oder Gewichtsstücke (it. $i$ pesi). Die dabei ausgeführten Handlungen sind das Auflegen einer Last (it. caricare il peso), das Wiegen/Wägen ${ }^{9} / \mathrm{Abwiegen} \mathrm{zum} \mathrm{Feststellen} \mathrm{des}$ Gewichts eines Körpers (it. pesare, ponderare) sowie das Abnehmen der Last (it. sollevare il peso). Auch das Schätzen von Gewichten gehört dazu. In Verbindung mit Gewicht werden dabei im Deutschen u. a. die Adjektive leicht (it. legg[i]ero, lieve) bzw. schwer (it. pesante) verwendet. Im Italienischen unterscheidet man zusätzlich das Adjektiv grave (,schwer'), das allerdings in Verbindung mit peso hauptsächlich in bildhafter Bedeutung verwendet wird (un grave peso $=$,eine schwere Belastung').

Seit dem Mittelalter wird Gewicht (nur im Singular) auch in einer weiteren, allgemeinen Bedeutung verwendet (3):

(3) Bedeutung, die im Verhältnis zu anderen Fakten schwerer wiegt und den Charakter eines Zusammenhangs oder Sachverhalts beeinflusst (z. B. das Gewicht einer Stimme innerhalb einer Partei; das Gewicht eines Staates) ${ }^{10}$. (Brockhaus Enzyklopädie online s. v. Gewicht/allgemein)

Hinsichtlich der Verwendung im Singular oder im Plural liegen je nach Bedeutung bzw. Verwendungszusammenhang gewisse Restriktionen vor. Während Gewicht gemäß den Bedeutungen (1a) und (1b) sowie (3) nur im Singular gebraucht wird, benutzt man das Wort in der unter (2) angegebenen Bedeutung (,Metallstücke, die als Maßeinheit beim Wiegen dienen') vorwiegend im Plural.

\subsection{Standard- und fachsprachlicher Gebrauch}

Spezifische Gewichtsbezeichnungen gibt es in der Standardsprache z. B. im Zusammenhang mit dem Gewicht von Personen (Körper-/Übergewicht), die auch in der Medizin verwendet werden. Darüberhinaus verwendet man viele Bezeichnungen in Fachgebieten wie z. B. der Wirtschaft (dem Handel), den Naturwissenschaften (Chemie, Mathematik und Physik) oder in

\footnotetext{
${ }^{9}$ Zur Etymologie und Wortfamilie der Verben wiegen und wägen cf. DWDS s. v. wägen.

${ }^{10}$ Diese Bedeutungserklärung (3) ist im Wortlaut identisch mit jener, die auf Duden online abrufbar ist. Zu der letztgenannten gehören folgende Beispiele: , , [...] in der Partei hat ihre Stimme großes, kein Gewicht; [...] dieses Land bekommt immer mehr Gewicht; [...] mit dem ganzen Gewicht seiner Persönlichkeit“. (Duden online s. v. Gewicht).
} 
der Philosophie, der Theologie, der Rechtsprechung und Verwaltung sowie im Sport (siehe Kapitel 3.1) und vereinzelt in besonderen Fachgebieten (siehe Kapitel 2.2.5). Im Zusammenhang mit dem technisch-wissenschaftlichen sowie dem kommerziellen, i. e. dem überprüfbaren Messbereich, entstanden im Deutschen (und Italienischen) zunächst Bezeichnungen zum Feststellen von ,Gewicht' (im Sinne der Bedeutungen ((1a) und (2); in Kapitel 2.1). Im Laufe der Zeit wurden mit einigen dieser Termini Ableitungen gebildet, oder es fanden Bedeutungserweiterungen statt, sodass sich die Verwendung der Bezeichnungen auf andere Gebiete erstreckte wie z. B. die Philosophie und Theologie oder das Rechtswesen und auf die Standardsprache. Dank semantischer Mutationen referiert man dementsprechend auf ein gefühltes ,Gewicht', das nicht objektiv messbar ist, weil es auf persönlichen Erfahrungen und auf Einschätzungen beruht (siehe Bedeutung (3) in Kapitel 2.1). Die folgenden Abschnitte stellen den Gebrauch von Gewicht (it. peso) in den unterschiedlichen Gebieten dar.

\subsubsection{Medizin}

In der Medizin wird auf „Gewicht“ mithilfe einiger Fachbegriffe Bezug genommen, die auch zur Standardsprache gehören. Dazu gehört neben Körpergewicht (auch einfach: Gewicht) und Über-/Untergewicht (i. d. Pathologie auch in Verbindung mit stark oder krankhaft) das Kompositum Gleichgewicht (it. equilibrio), das in der Medizin definiert wird als: „die über das Gehirn, speziell Kleinhirn, kontrollierte Körperlage im Verhältnis zum Raum und zur Schwerkraft.“ (Cf. Hammerschmid-Gollwitzer 1981: 154; Pera/Schmiedebach 2010: 109). Davon leiten sich weitere Fachtermini wie Gleichgewichtsstörung (it. disturbo dell'equilibrio), Gleichgewichtsempfinden (senso dell'equilibrio) und Gleichgewichtsorgan (organo dell'equilibrio) ab. ${ }^{11}$ Das nominale Kompositum Gleichgewichtsstörung bedeutet eine:

mangelhafte bis fehlende, bewußtwerdende Kontrollfähigkeit über die Körperlage mit Störung des Gleichgewichtsempfindens. Vorkommen bei Kleinhirnkrankheiten, Erkrankungen des Innenohrs und Vestibularapparates [...] und verschiedenen Hirnerkrankungen [...].

(Hammerschmid-Gollwitzer 1981: 154)

\subsubsection{Wirtschaft und Handel}

Im internationalen Handel werden vor allem Komposita mit Gewicht als Erst- oder Letztglied mit und ohne Fugenelement in der Bedeutung (1a) verwendet wie: Gewichtangabe ${ }^{12}$

\footnotetext{
${ }^{11}$ Die hier in Klammern angegebenen italienischen Entsprechungen stammen fast ausschließlich aus dem zweisprachigen Wörterbuch Giacoma/Kolb (2019); in Verbindung mit dem Grundwort Gleichgewicht wird in den konsultierten zweisprachigen deutsch-italienischen Wörterbüchern außerdem das nominale Kompositum Gleichgewichtssinn aufgeführt (cf. ibd. und Giacoma/Kolb 2016), für das es jedoch im einsprachigen Wörterbuch DWDS keine Bedeutungsangaben gibt, sondern für Gleichgewichtsempfinden (cf. DWDS s. v. Gleichgewichtsempfinden vs. s. v. Gleichgewichtssinn). Eine Korpusrecherche deutschsprachiger Texte (ohne Wikipedia) im Zeitraum 1950/1960 - 2020 ergab in diesem Zusammenhang folgende Treffer: Gleichgewichtssinn, 1950-2020 = 2.998 Treffer; Gleichgewichtsgefühl, 1960-2020 = 547 Treffer und für Gleichgewichtsempfinden, 1960-2020 = 26 (!) Treffer (cf. DeReKo s. v. Gleichgewichtssinn (ibd.); s. v. Gleichgewichtsgefühl (ibd.); s. v. Gleichgewichtsempfinden). Somit ist Gleichgewichtssinn - zumindest in vielen deutschsprachigen Medientexten - die geläufigere Bezeichnung.

${ }^{12}$ Nebenform: mit Fugenelement (cf. DWDS s. v. Gewichtsangabe).
} 
(indicazione f del peso [Z], Gewichtbescheinigung (kein Eintrag) ${ }^{13}$, Gewichtskontrolle (controllo $\mathrm{m}$ del peso [Z]); Brutto-/Nettogewicht (it. peso lordo/netto [Z]; [ZS] ${ }^{14}$ ), Gesamtgewicht (peso m complessivo/totale [Z]) u. v. a. (siehe Kapitel 3.1.1). Für die dabei üblichen Komposita mit Gewicht als Erst- und als Letztglied gibt es im Italienischen Mehrwortentsprechungen mit peso im Singular und teilweise auch im Plural (ibd.). In der italienischen Wirtschafts-(bzw. Handels-)sprache und im Verkehrswesen spricht man dagegen von portata oder carico (z. B. ,(zulässiges) Ladegewicht', it. portata $f$ (massima)/(massimo) carico consentito; cf. Giacoma/Kolb 2019: 659). Ein anderer Ausdruck der italienischen Handelssprache ist peso d'origine (cf. Treccani Vocabolario online s. v. péso ${ }^{2}$ ), das im Deutschen auch als Originalgewicht bezeichnet wird (cf. Sieveking 2019: 46), wobei diese Bezeichnung polysem ist, denn damit kann auch ein ,geeichtes Gewichtsstück, das als Normalmaß gilt" gemeint sein (DRW s. v. Originalgewicht). Die Bezeichnung ist in den hier konsultierten Wörterbüchern nicht gelistet (cf. Duden 2019; Duden online; DWDS; Giacoma/Kolb 2019, 2016; Troike Strambaci 2009). ${ }^{15}$

\subsubsection{Naturwissenschaften (Physik, Mathematik, Chemie)}

Neben Länge und Zeit ist ,Gewicht` eine der drei Basisgrößen, zu deren Beschreibung Maßzahlen und Maßeinheiten verwendet werden. In der Physik sind die entsprechenden Fachtermini durch das Internationale Einheitensystem für physikalische Größen (SI) exakt definiert. Dabei wird jeder Größe eine sogenannte Basiseinheit zugeordnet. Für ,Gewicht‘ ist es das Ki$\operatorname{logramm}(\mathrm{kg})$. Ein Gramm entspricht einem Tausendstel eines Kilogramm und eine Tonne 1000 Kilogramm.

Die Komplexität der Bedeutungszuweisung von Gewicht wird anhand des folgenden Zusammenhangs deutlich: Während die Masse eines Körpers ortsunabhängig und somit unveränderlich ist, hängt das Gewicht eines Körpers von der von Ort zu Ort variierenden Gravitationsbeschleunigung ab, die auf ihn einwirkt. Aus wissenschaftlicher Sicht, i. e. in den Naturwissenschaften (Physik), ist der in der deutschen Allgemeinsprache verwendete Begriff Gewicht somit fachlich nicht korrekt, und es muss entsprechend Gewichtskraft heißen (it. forza peso). ${ }^{16}$

\footnotetext{
${ }^{13}$ In den hier konsultierten Wörterbüchern fanden sich dafür keine italienischen Entsprechungen (cf. Giacoma/Kolb 2016 [ZS]; 2019 [Z]). Im folgenden Text wird darauf (kein Eintrag) mittels Abkürzung „KE“ (kein Eintrag) verwiesen.

${ }^{14}$ Bei den hier konsultierten zweisprachigen Wörterbüchern handelt es sich um die beiden Ausgaben von Giacoma/Kolb (2019; [Z]) und (2016; (ZS]).

${ }^{15}$ Im Italienischen bedeutet diese Bezeichnung: ,p. d'origine ( $o$ all'origine), il peso della merce nel luogo o magazzino del venditore e all'epoca della spedizione al compratore“. (Treccani Vocabolario online s. v. péso ${ }^{2}$ ).

16 „Gewicht [...]: heute nicht mehr übliche Bezeichnung für die Gewichtskraft, die Kraft G, mit der ein Körper in Richtung Erdmittelpunkt hingezogen wird. Die Gewichtskraft $\mathrm{G}$ ist einerseits von der Masse $\mathrm{m}$ des Körpers, andererseits von der Erdbeschleunigung g abhängig. Es gilt: $G=\mathrm{m} \cdot \mathrm{g}$. Maßeinheiten der Gewichtskraft sind die Einheiten der Kraft.“ (Cf. Brockhaus Enzyklopädie online s. v. Gewicht „Physik“; „Formelzeichen FG, SI-Einheit ist das Newton (N); die Kraft, die ein Körper im Schwerefeld eines Himmelskörpers (z. B. der Erde) zu dessen Mittelpunkt hin erfährt. Die Gewichtskraft berechnet sich aus dem Produkt der Fallbeschleunigung g und der Masse m: [...]. „Die Gewichtskraft ist aufgrund der veränderlichen Gravitations- und Zentrifugalkräfte im Gegensatz zur Masse eine ortsabhängige physikalische Größe [...].“ (Brockhaus Enzyklopädie online s. v. Gewichtskraft/Physik).
} 
In der Mathematik (Statistik) hat Gewicht die folgende Bedeutung (4):

Faktor, durch den eine Größe gegenüber anderen bei der Mittelwertbildung stärker oder schwächer berücksichtigt wird (z. B. wird man ein Messergebnis mit einem kleinen relativen Fehler stärker „gewichten“ als ein Messergebnis mit einem größeren relativen Fehler). Den so durch Gewichtung erhaltenen Mittelwert nennt man gewogenes (gewichtetes) Mittel [...].

(Brockhaus Enzyklopädie online s. v. Gewicht/Mathematik)

Auch im Italienischen spricht man in diesem Fachgebiet (i. e. Statistik) von peso: ,[...] In statistica, denominazione dei coefficienti che si attribuiscono ai singoli termini di una serie di dati numerici o di misure in relazione alla loro importanza o frequenza". (Treccani Vocabolario online s. v. péso ${ }^{2}$ ).

Der im Italienischen in der Analytischen Chemie geläufige Ausdruck portare a peso (costante) (wörtlich: ,auf ein (konstantes) Gewicht bringen') verweist auf ein Verfahren der quantitativen Analyse, das im Deutschen unter der Bezeichnung Darr-(Wäge)-Methode/Verfahren bzw. Gewichtsanalyse bekannt ist (cf. Brockhaus Enzyklopädie online s. v. Gravimetrie). Dabei wird eine Probe/Substanz bis zur Gewichtskonstanz (it. peso costante) getrocknet. Mittels Wäge in der Bezeichnung der Methode bzw. des Verfahrens besteht auch im Deutschen ein entfernter Bezug zum Wortfeld, Gewicht'.

\subsubsection{Philosophie und Theologie}

Philosophische und theologische Schriften behandeln die Größe ,Gewicht` im objektiv nachprüfbaren und im bildhaften Sinne. Demokrit (460 - 370 v. Chr.) führte das Gewicht wegen seiner Relevanz für Atome als Größe ein und Epikur (341 - 271 v. Chr.) spricht in seiner Atomistik neben Größe und Gestalt von einer dritten Grundeigenschaft fester Körper und verwendet dafür den Begriff báros (griech. ,Schwere, Druck'; cf. Horn/Rapp 2008: 76; 81).

Das Alte Testament nimmt an mehreren Stellen Bezug auf Gewicht in seinen unterschiedlichen Bedeutungen (siehe Kapitel 2.1) wie z. B. in: „Du sollst nicht zweierlei Gewicht, groß und klein, in deinem Beutel haben.“ (5. MOSE 25.13); „Du sollst ein volles und rechtes Gewicht und ein volles und rechtes Maß haben, auf da[ss] dein Leben lange währe [...]" (5. MOSE 25.15); „Zweierlei Gewicht ist dem HERRN ein Greuel und eine falsche Waage ist nicht gut.“ (Sprüche 20.23); „Aber du hast alles nach Maß, Zahl und Gewicht geordnet.“ (Weis. Sal. 11.21). Der Grundsatz „[Gott hat] alles nach Maß, Zahl und Gewicht [geordnet]“ (ibid.) unterstreicht die Bedeutung der Basisgröße „Gewicht“. Bibelzitate aus dem Neuen Testament, in denen Gewichtsbezeichnungen vorkommen, sind u. a.:

sein Pfund vergraben $=$ seine Gaben nicht anwenden (nicht zur Geltung bringen); [...] in Anlehnung an ein Wort des Evangelisten Matthäus $(25,18)$ in Luthers Übersetzung. Gegensatz: [...] mit seinem Pfunde wuchern = seine Gaben erfolgreich benutzen, [...] in Anlehnung an ein Wort des Evangelisten Lukas (19: 12ff.) in Luthers Übersetzung [...]; Einer trage des anderen Last! [...] Paulus [, Galaterbrief,] 6,2).

(Mackensen 1981: 656; 229; Hervorhebungen i. O.)

\subsubsection{Rechtswesen und andere Fachgebiete}

Bereits in der Antike wurden Bedeutungsableitungen von Gewichtsbezeichnungen geprägt, um damit in der Rechtssprache bildhaft auf bestimmte juristische Zusammenhänge und 
Rechtskonzepte zu verweisen. In der juristischen Terminologie gibt es den aus dem Lateinischen stammenden Ausdruck numero et pondere (,Zahl und Gewicht'), der von lat. pondus (,Gewicht, Last; römisches Pfund“) abgeleitet ist und für „vertretbare“ Sachen steht, die man „nach Zahl und Gewicht bestimmt“ (Preuß 2009: 829).

In juristischen Verfahren spricht man außerdem vom sogenannten Gewicht der Beweise. Damit bezeichnet man Beweise, mit denen die Schuld oder Verantwortung eines Angeklagten für eine Tat mithilfe gewichtiger Beweise nachgewiesen wird. Aus dem Wortfeld ,Gewicht' stammt auch der Ausdruck Last der Anklage. Die Anklage wird bildhaft als schweres Gewicht verstanden, die vor Gericht erhoben wird. In der deutschen Rechtssprache leiten sich Fachbegriffe von dieser Bedeutungsübertragung ab wie die Beweislast (it. onere della prova). Es handelt sich dabei um ,Anschuldigungen, die auf einer Person lasten“ (accuse che gravano su una persona). Die Aussage eines Zeugen dient entweder der Belastung oder Entlastung eines Beschuldigten und kann ihn somit be- oder entlasten (incriminare/scagionare l'imputato; Troike-Strambaci/Helffrich 2009: 63).

In der Jägersprache hat Gewicht im Deutschen schließlich noch die im Frühneuhochdeutschen entstandene Bedeutung (5): „Ein sehr starkes Geweih heißt auch Gewicht“ (Lenz 1873: 492493). Diese Erklärung ist im Wortlaut identisch mit dem Eintrag im Etymologischen Wörterbuch (cf. Kluge/Götze 2011: 357). Im Italienischen lautet diese Entsprechung (im Sinne von ,Geweih‘) „corna, f. pl.“(Giacoma/Kolb 2019: 455).

In der Ausgangsbedeutung, objektiv festgestelltes Gewicht‘ ist ursprünglich das feminine Substantiv wicht (e) belegt. Heute gilt das Nomen Wichte als veraltend und wird in der fachsprachlichen Bedeutung (,spezifisches Gewicht') verwendet (cf. DWDS s. v. Wichte). Es stammt von mittelniederdeutsch wicht(e) (siehe Kapitel 2.1). ${ }^{17}$

Das Adjektiv wichtig wiederum ist eine Ableitung von Gewicht und seit dem 14. Jh. belegt. In seiner ursprünglichen Form gewichtig bezog man sich damit auf ein ,objektiv festgestelltes (großes) Gewicht'. Erst später entwickelten sich die übertragenen Bedeutungen, mit denen wir wichtig und seltener gewichtig heute im Deutschen verwenden: ,bedeutsam; ernst; bedeutend; wichtig' (cf. DWDS s. v. gewichtig).

In Tabelle 1 ist die Bedeutungsentwicklung des Wortes Gewicht und seiner adjektivischen Ableitung (ge-)wichtig dargestellt:

\footnotetext{
17 „Gewicht n. ,Schwere, Bedeutsamkeit‘. Die Präfixbildung ahd. Giwihti (um 1100), mhd. gewiht(e), mnd. gewichte, mnl. ghewichte, nl. gewicht setzt ein im Hd. nicht bezeugtes, aber in mnd. wicht(e) f., mnl. nl. wicht, aengl. wiht, engl. weight, anord. Veett erhaltenes Abstraktum germ. *wehti- f. ,Gewicht' zu dem unter wägen (s. d.) behandelten Verb voraus. Gewichtig Adj. ,schwerwiegend, maßgeblich` (15. Jh.). Übergewicht n. ,zuviel Gewicht‘ (16. Jh.)“ (DWDS, s. v. Gewicht). „Gewicht: Mhd. gewiht(e) ist eine ge-Bildung zu dem im Hochd. untergegangenen Substantiv mniederd. wicht ,Gewicht, Schwere' [...]“" (Duden 2020b: 323; Hervorhebungen i. O.).
} 


\begin{tabular}{|c|c|c|}
\hline WORT & AUSGANGSFORM UND -BEDEUTUNG & ABGELEITETE BEDEUTUNG \\
\hline Gewicht (Sn) & $\begin{array}{c}\text { wicht(e) (Archaismus); früher: ,objektiv fest- } \\
\text { gestelltes Gewicht'; heute: , spezifisches Ge- } \\
\text { wicht }^{\star}\end{array}$ & ,Bedeutung, Wichtigkeit \\
\hline wichtig (Adj.) & $\begin{array}{c}\text { gewichtig; früher: bezogen auf ein objektiv } \\
\text { festgestelltes Gewicht }\end{array}$ & (ab 1600): , bedeutend, ernst, wichtig \\
\hline
\end{tabular}

Tabelle 1: Bedeutungsentwicklung von Gewicht und (ge-)wichtig

\subsection{Intersprachliche Phänomene}

Im Zusammenhang mit Gewicht/peso gibt es neben den standard- und fachsprachlichen Bedeutungen auch eine Vielzahl von Ableitungen, Wortzusammensetzungen sowie Wortkombinationen (i. e. Wortverbindungen und Redewendungen; siehe Kapitel 3.2.1 und 4), für die sich in den beiden Sprachen je nach Verwendungszusammenhang jeweils unterschiedliche Entsprechungen entwickelt haben.

So verwendet man für das deutsche Nomen Gewicht im Singular und in Wortbildungen im Italienischen nicht ausschließlich peso $^{18}$, sondern je nach Bedeutung und Kontext auch carico, massa oder portata bzw. peso im Plural (pesi). Der Bedeutungszusammenhang, der im Deutschen zwischen Gewicht und Bedeutung besteht, kann ebenfalls zu falschen Schlüssen führen (siehe Bedeutung (3) in Kapitel 2.1). Im Italienischen lauten die Entsprechungen von Bedeutung in den hier konsultierten Wörterbüchern neben peso (,Gewicht') und importanza (,Bedeutung, Wichtigkeit, Gewicht') auch influenza oder prestigio (,Einfluss` bzw. ,Ansehen`) (siehe Kapitel 2.1; 2.2.4; 2.2.5; 3.1; 3.1.1; 3.2.1; 3.3).

Im Italienischen kann peso - ebenso wie Gewicht im Deutschen - im übertragenen Sinne auch ,Bedeutung, Wert' bedeuten. Dagegen haben einige Derivationen wie z. B. pesante oder pesantezza nicht die Bedeutungen und Verwendungen, die die Derivationen von Gewicht (ge-)wichtig, Gewichtung/gewichten ${ }^{19}$ oder Wichtigkeit haben (siehe oben in diesem Kapitel und in Tabelle 1, Kapitel 2.2.5). Die Synonyme von Bedeutung (Gewicht und Wichtigkeit) in Verbindung mit einer Polysemie von Bedeutung (,Wichtigkeit' und ,Wortbedeutung'; it. significato) können deshalb bei Nicht-Muttersprachlern zu einer gewissen Verwirrung führen.

In der italienischen Umgangssprache wird peso auch adjektivisch verwendet, fast als Synomym für pesante, sodass es für peso noch eine Bedeutung (6) gibt (16. Jh.: ursprünglich regionaler Ausdruck aus der Toskana, siehe auch: ,andar giù peso, 'di schianto, di brutto, senza potersi né trattanere né aiutare'. Fig. 'noioso, opprimente, insopportabile', quant'è peso quel ragazzo!“; Devoto et al. 2017: 1588). Die deutschen Entsprechungen für peso in dieser Ver-

\footnotetext{
18 „Peso: 1) s. m. [sec. XIV] - forza di gravità, misura adottata nelle operazioni commerciali, carico” (Nocentini/Parenti (2010: 880).

19 „1. das Gewichten (1); das Gewichtetwerden, [...:] eine Gewichtung von Daten vornehmen; 2. das Gewichten (2); Festlegung von Schwerpunkten“ (Duden online s. v. Gewichtung); „1. von etwas unter Berücksichtigung der Häufigkeit des Auftretens einzelner Werte einen Durchschnittswert bilden und damit den Wert, die Bedeutung der einzelnen Größen einer Reihe ermitteln; 2. die Bedeutung, Bedeutsamkeit, Wichtigkeit von etwas festhalten, festlegen; Schwerpunkte bei etwas setzen, [...:] diese Pläne gilt es nun [neu, richtig] zu gewichten“ (Duden online s. v. gewichten).
} 
wendung (,unerträglich, langweilig; lästig' ${ }^{20}$ haben somit keinen Bezug zur Wortfamilie, sondern nur eingeschränkt zum Wortfeld, Gewicht‘ (lästig).

Bei den genannten Aspekten handelt es sich um lexikalische, semantische und phraseologische Phänomene, die im deutsch-italienischen Sprachkontakt berücksichtigt werden müssen. Eine Einteilung der Bezeichnungen nach lexikalischen Kriterien kann hilfreich sein und dafür sorgen, dass man je nach Bedarf eine passende Entsprechung in der einen bzw. der anderen Sprache findet. Diese Darstellung ist außerdem faszinierend, denn sie erfolgt auf drei Ebenen:

1. Systematische Darstellung deutscher Gewichtsbezeichnungen (inkl. Derivationen, Komposita, Wortverbindungen, Redewendungen);

2. italienische Entsprechungen von 1.;

3. italienische Übersetzungen ausgewählter deutscher Gewichtsbezeichnungen.

\section{Deutsche Gewichtsbezeichnungen und italienische Entsprechungen}

Die Komplexität intersprachlicher Übereinstimmungen und Differenzen im Zusammenhang mit Gewichtsbezeichnungen wird anhand einer Darstellung der Wörterbucheinträge von Gewicht und einiger verwandter Bezeichnungen im zweisprachigen italienisch-deutschen Wörterbuch deutlich:

Gewicht $-[\ldots] 1<$ nur [Singular]> (gewogene Schwere) (+FAHRZEUG, GEGENSTAND, PERSON) peso $\mathrm{m}[\ldots] 2<$ nur [Singular] $>$ [Physik] peso $\mathrm{m} 3<$ nur [Singular] $>$ (Bedeutung) peso $\mathrm{m}$, importanza f $4<$ meist [Plural] $>$ (Metallstücke zum Wiegen) peso $\mathrm{m} 5<$ meist [Plural] $>$ [Sport] peso $\mathrm{m}[\ldots]$ großes/geringes [Gewicht] haben avere $[\ldots] \mathrm{molto} /($ un) gran $[\ldots] /($ poco) peso, avere molta/poca importanza; [Gewicht] haben (ARGUMENT, MEINUNG), avere peso/importanza; $[\ldots]$ ins $[$ Gewicht $]$ fallen $[\ldots]$, avere $[\ldots]$ molto peso[...]/[molta importanza], essere importante; auf [etwas] [...] [Gewicht] legen, [etwas] [...] [Gewicht] beimessen [...] dare peso/importanza a qc; [...] sein ganzes [Gewicht] (für jdn/etw) in die Waagschale werfen, usare/[far valere] [...]tutta la propria influenza[...],/[tutto il proprio prestigio] (per qu/qc);

Gewichtheben $-[\ldots]$ sollevamento $\mathrm{m}$ pesi, pesistica $\mathrm{f}$

Gleichgewicht - [...] 1 (Stabilität) equilibrio m 2 (innere Ausgeglichenheit) equilibrio m; gewichtig - [...] 1 (bedeutend) [...] di un certo peso, importante; $[\ldots]$ autorevole, di prestigio $[\ldots]$

Gewichtung $-[\ldots]$ valutazione f;

Wichte-KE ;

Wichtigkeit $-[\ldots]$ importanza f;

Wucht $-[\ldots]$ forza $\mathrm{f}$, violenza $\mathrm{f}$, impeto $\mathrm{m} ;[$...] das ist eine [Wucht]![...], è una cannonata! [...] sie ist eine $[$ Wucht $]$ ! [...]. lei è uno schianto! [...].

(Giacoma/Kolb 2019: 455; 1299; 1322; (Hervorhebungen i. O.))

Im zweisprachigen deutsch-italienischen Wörterbuch unterscheidet man demnach fünf Bedeutungen von Gewicht, i. e. in standard- und fachsprachlichen Verwendungen 1), gewogene Schwere von Gegenständen, Fahrzeugen oder Personen`; 2) Naturwissenschaften

20 „Adj. ,belastend, beschwerlich, störend, unangenehm‘, Ableitung des 15. Jhs. von Last.“ (Cf. DWDS s. v. lästig). 
(Physik); 4) ,Metallstücke zum Wiegen“ und 5),Gewichtsklassen` u. a. im Sport. In einer übertragenen Bedeutung werden italienische Entsprechungen von Gewicht wie im Deutschen unter (3) angegeben. Der Übersicht halber sind die im einsprachigen deutschen (linke Spalte: Gewicht: DWB) sowie die im zweisprachigen deutsch-italienischen Wörterbuch angegebenen Bedeutungen (rechte, breite Spalte: Gewicht: D-I WB) mit den entsprechenden Zahlen sowie ihrer Anzahl (siehe letzte Zeile) in Tabelle 2 dargestellt:

\begin{tabular}{|c|c|c|c|}
\hline \multirow{6}{*}{ 更 } & Gewicht: D & \multicolumn{2}{|c|}{ Gewicht: D-I WB } \\
\hline & 1a) & 1 & $\begin{array}{r}<\text { nur }[\text { Singular }]>(\text { gewogene } \text { Schwere })(+\mathrm{FAHRZEUG}, \mathrm{GE}- \\
\text { GENSTAND, PERSON }) \text { peso } \mathrm{m}[\ldots]\end{array}$ \\
\hline & 1b) & 2 & $<$ nur $[$ Singular $]>[$ Physik] peso $\mathrm{m}$ \\
\hline & 2) & 4 & $<$ meist [Plural] $>$ (Metallstücke zum Wiegen) peso m \\
\hline & 2) & 5 & $<m e i s t[$ Plural $]>[$ Sport $]$ peso $\mathrm{m}[\ldots]$ \\
\hline & 3) & 3 & $<$ nur $[$ Singular $]>($ Bedeutung $)$ peso $\mathrm{m}$, importanza $\mathrm{f}$ \\
\hline Anzahl & 4 & & 5 \\
\hline
\end{tabular}

Tabelle 2: Bedeutungen von in ein- und zweisprachigen Wörterbüchern (WB)

Die im einsprachigen deutschen Wörterbuch zugewiesene Bedeutung (1b) von Gewicht (cf. Duden online s. v. Gewicht) hat im zweisprachigen deutsch-italienischen Wörterbuch demnach die Bedeutung (2); die im Sport verwendeten Gewichte und die beim Wiegen eingesetzten Gewichtsstücke, die laut einsprachigem deutschen Wörterbuch die Bedeutung (2) haben, stehen im zweisprachigen deutsch-italienischen Wörterbuch in den Bedeutungen (4) bzw. (5). ${ }^{22}$

Die folgenden Kapitel $(3.1 ; 3.1 .1 ; 3.2 ; 3.2 .1 ; 3.3 ; 4.1 ; 4.2)$ sind der kontrastiven Gegenüberstellung einfacher und komplexer deutscher Gewichtsbezeichnungen und ihren italienischen Entsprechungen gewidmet. Es handelt sich dabei um Lexeme, Wortverbindungen und Redewendungen mit Gewicht inkl. Ableitungen oder Komposita, wie sie in Wörterbüchern, Medientexten und Übersetzungsportalen verzeichnet sind.

\subsection{Lexeme}

In diesem Abschnitt werden deutsche Gewichtsbezeichnungen (i. e. Einträge mit den Wortbestandteilen Gewicht-/-gewicht- gemäß den Wörterbüchern Duden online und DWDS) und ihre italienischen Entsprechungen in ein- und zweisprachigen Wörterbüchern (Treccani Vocabolario online); [T]; Giacoma/Kolb 2019 [Z]; 2016 [ZS]; einschlägigen Nachschlagewerken zu besonderen Fachgebieten [G]) dargestellt. ${ }^{23}$ Dabei wurden sowohl die unterschiedlichen

${ }^{21}$ Die verwendeten Abkürzungen bedeuten: $\mathrm{D}=$ deutsch (einsprachig); D-I=deutsch-italienisch; WB=Wörterbuch. Die Bedeutungen von „Gewicht“ (1a); (1b); (2) und (3) gemäß einsprachigen deutschen Wörterbüchern bzw. Nachschlagewerken stehen in Kapitel 2.1.

${ }^{22} \mathrm{Im}$ Deutschen lautet die Bedeutung von Gewicht in dieser Verwendung: „2. Körper von bestimmter Schwere [...] Beispiele [...] (Sport) ein Gewicht stemmen“; cf. Duden online s. v. Gewicht).

${ }^{23}$ Es handelt sich dabei um Einträge mit den Wortbestandteilen Gewicht-/-gewicht- der einsprachigen Wörterbücher Duden online sowie DWDS. 
Bedeutungen von Gewicht berücksichtigt (i. e. (1a), (1b), (2) und (3); siehe Kapitel 2.1) als auch der standard- bzw. fachsprachliche Gebrauch, i. e.: Standardsprache (Sspr.)/Medizin; Wirtschaft/Handel/Verkehrswesen/Mechanik; Naturwissenschaften; Sport/u. a., Sspr./Theologie/Philosophie/Rechtswesen/u. a. (siehe Kapitel 2.2.1-2.2.5).

Als erstes werden die italienischen Entsprechungen in der Gruppe A) Einfaches Lexem: Gewicht dargestellt, gefolgt von jenen in der Gruppe B) Gewicht[s]-/gewicht- als Erstglied in Komposita; Gewicht/gewicht- in Derivationen; in der Gruppe C) -gewicht als Letztglied in Komposita und schließlich der Gruppe D), in der -gewicht- als Binnenglied in komplexen Wortbildungen (Komposita, Derivationen und Mischformen) vorkommt. ${ }^{24}$

\subsubsection{Lexeme (einfache Lexeme, Komposita, Derivationen, Mischformen))}

A) Einfaches Lexem: Gewicht (abgekürzt: G./g.)

1a) Sspr./Medizin; Wirtschaft/Handel/Verkehrswesen/Mechanik: „(nur im Singular) peso m“ ([Z]; [ZS]);

1b) Naturwissenschaften: „(nur im Singular) - peso m“ ([Z]; $[\mathrm{ZS}])$; forza peso $[\mathrm{T}]$;

2) Sport/u. a.: „,[meist Plural] - peso m“ ([Z]; [ZS]); Wirtschaft/Handel u. a. (,Gewichtsstücke'): peso ([Z]; [ZS]); pesi; massa [T];

3) Sspr./Theologie/Philosophie/Rechtswesen/u. a.: peso $\mathrm{m} /$ importanza $\mathrm{f}$ ([Z]; [ZS]).

B) Gewicht[s]-/gewicht- als Erstglied in Komposita (G./g.); Gewicht/gewicht- als Erstglied in Derivationen:

1a) Sspr./Medizin: gewichtig - ,scherz (korpulent) (Person) grosso“ ([Z]; [ZS]); G.[s]abnahme - „calo m/dimininuzione f di peso“ ([Z]); G.[s]bestimmung - KE; G.[s]problem - ,problema m di peso“25 ([Z]); G.[s]verlagerung - „1 spostamento m del peso“ ([Z]); G.[s]verlust - ,perdita f di peso“ ([Z]); G.[s]zunahme - ,aumento m di peso“ ([Z]); Handel/Verkehrswesen: G.[s]abweichung - KE; G.[s]angabe - ,indicazione f del

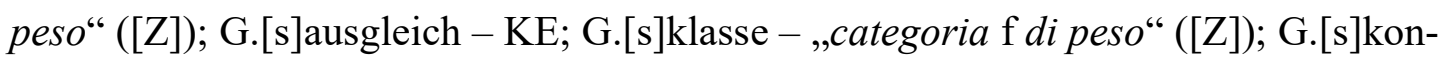
trolle - ,controllo m del peso“ ([Z]); G.[s]verlust - ,perdita f di peso“ ([Z]);

1b) Naturwissenschaften: gewichten - KE; G.[s]ausgleich - KE; Gewichtung - KE; G.[s]bestimmung - KE; G.[s]einheit - „unità f di peso“ ([Z]); Gewichtung - KE;

2) Sport: G.heben - pesistica, sollevamento pesi ([Z]); G.heber/-in - ,pesista; sollevatore/-trice di pesi“ ([Z]); G.[s]klasse - categoria di peso ([Z]); G.[s]verlagerung - „1 spostamento $\mathrm{m}$ del peso" ([Z]);

3) Sspr./Theologie/Philosophie/Rechtswesen/u. a.: gewichten ${ }^{26}-,,<$ ohne ge-> [transitiv, gehoben] etw [gewichten] soppesare qc [...]“ ([Z]); gewichtig - „1 [gehoben]

\footnotetext{
${ }^{24}$ Einige der in den Kapiteln 2.1; 2.2.1-2.2.5 und 2.3 genannten Gewichtsbezeichnungen und ihre italienischen Entsprechungen, werden hier nicht zum wiederholten Mal aufgeführt.

25 Im deutsch-italienischen Wörterbuch von Giacoma/Kolb (2019) steht bei dem Eintrag Gewichtsproblem der Zusatz „,<meist pl>“. Eine Korpusanalyse, basierend auf Medientexten deutscher Medien wie ARD, Dlf oder ZDF, konnte dies nicht bestätigen. In Wortverbindungen wird das Kompositum auch im Singular verwendet wie z. B. in: ein G.-problem haben oder jmds./das G.-problem lösen/in den Griff bekommen, gegen das/jmds. G.-problem kämpfen u. a.

26 Bedeutungserklärung: „1. [Statistik] von etw. unter Berücksichtigung der Häufigkeit des Auftretens einzelner Werte einen Durchschnittswert bilden und damit den Wert, die Bedeutung der einzelnen Größen einer Reihe
} 
(bedeutend) (ARGUMENT, GRUND, PROBLEM) di un certo peso; importante; (MANN, PERSÖNLICHKEIT) auch autorevole; di prestigio“ [Z]; „1 geh (wichtig) \{ARGUMENT\} di un certo peso, importante; \{PERSÖNLICHKEIT\} auch autorevole, di prestigio“ [ZS]; Gewichtigkeit - KE; Gewichtung - valutazione ([Z]; [ZS]); Gewichtsverlagerung - „3 spostamento del baricentro“ ([Z]).

C) Letztglied ${ }^{27}$ : -gewicht (,g. $\left.{ }^{6}\right)$ :

1a) Sspr./Medizin(7): Gleichg. - equilibrio m ([Z]); [ZS]), (siehe 2.2.1); Idealg. - ,p. m forma/ideale“ $([\mathrm{Z}])^{28}$; Körperg. - ,p. m corporeo“ ([Z]; [ZS]); Normalg. - ,p. m normale“ ([Z]); Traumg. - ,p. m ideale“ ([Z]); Überg. - sovrappeso ([Z]; [ZS]); Unterg. - sottopeso $([\mathrm{Z}] ;[\mathrm{ZS}])$;

Wirtschaft/Handel/Verkehrswesen/Mechanik: Abtropfg. - KE; Bruttog. - p. m lordo ([Z]); [ZS]); Darrg. - KE (peso anidro ([T]; [G] $)^{29}$; Eigeng. ${ }^{30}-$, ,1 mech $(+\mathrm{LKW})$ tara f, 2 [Wirtschaft, Handel] (Nettogewicht) p. m netto“ ([Z)]; Gesamtg. - „,+FAHRZEUG) p. m complessivo/totale“ ([Z)]; Frischg. - KE; Füllg. ${ }^{31}$ - „1 [Wirtschaft,

ermitteln; 2. die Bedeutung, Bedeutsamkeit, Wichtigkeit von etw. festhalten, festlegen; Schwerpunkte bei etw. setzen“. (DWDS s. v. gewichten).

${ }^{27}$ Für einige Gewichtsbezeichnungen finden sich in den zweisprachigen Wörterbüchern keine italienischen Entsprechungen (siehe „KE“). Die unter B) ,-gewicht als Letztglied“ angegebenen italienischen E. stammen überwiegend - mit einigen wenigen Ausnahmen wie Gleichgewicht, Hauptgewicht, Ladegewicht oder Über/-Untergewicht und die Gewichtsklassen im Sport Mittel-/Schwergewicht (auch in Giacoma/Kolb 2016, [ZS]) - aus dem zweisprachigen deutsch-italienischen Wörterbuch Giacoma/Kolb (2019), [Z], (Giacoma/Kolb 2019: 455; 462; 1299; 1322; ohne Hervorhebungen i. O.; [Z]). Italienische Entsprechungen aus dem einsprachigen Wörterbuch Treccani sind jeweils mit [T] ausgewiesen und beziehen auf die Erläuterungen und Beispiel unter Eintrag peso ${ }^{2}$ (cf. Treccani Vocabolario online s. v. péso ${ }^{2}$; Eine italienische Entsprechung (peso anidro) (für deutsch: Darrgewicht) findet sich in Giordano (1999); [G]).

${ }^{28}$ Im Italienischen unterscheidet man laut Treccani zwischen peso forma (,quello di un atleta che sia al meglio della sua forma fisica") und peso ideale ("il peso corporeo considerato ottimale per una persona in base a determinati parametri, adottato come punto di riferimento nei calcoli dei fabbisogni calorici per la preparazione delle diete"). Dementsprechend heißt die italienische Entsprechung von Idealgewicht bei Sportlern also peso forma und allgemein bei Personen peso ideale (cf. Treccani Vocabolario online s. v. péso ${ }^{2}$ ).

${ }^{29}$ Die Bezeichnung Darrgewicht bedeutet laut Duden online: „Gewicht des Holzes in absolut trockenem Zustand“; sie ist in den hier konsultierten aktuellen zweisprachigen Wörterbüchern nicht verzeichnet (cf. Giacoma/Kolb 2019/2016). Im Italienischen wird dafür in fachsprachlichen Kontexten (Holz/Holzwirtschaft; i. e. Holztrocknung) die Bezeichnung peso $\mathrm{m}$ anidro (anidro $=$, privo di acqua') verwendet (cf. Treccani Vocabolario online s. v. anidro; Giordano 1999: 17; 22; [G]).

${ }^{30}$ Laut DWDS unterscheidet man folgende Bedeutungen: „1. eigenes Gewicht; 2. a) Technik: eigenes Gewicht ohne (Zu-) Ladung; b) Wirtschaft: Gewicht der Ware ohne Verpackung“. Außerdem wird das Wort in übertragener Bedeutung verwendet, z. B. in ,das politische Eigengewicht Europas“ (Duden online s. v. Eigengewicht; DWDS s. v. Eigengewicht). Im zweisprachigen Wörterbuch werden zwei italienische Entsprechungen dafür angegeben: „1 bezogen auf das Verkehrswesen - tara f“ (Giacoma/Kolb 2019: 32), wobei diese Bezeichnung im Deutschen wiederum folgende Bedeutungen hat: „Tara [italienisch, eigentlich ,Abzug für Verpackung“, von arabisch țarh ,Abzug'] die, -/...ren, Handel: 1) die Verpackung einer Ware; 2) Taragewicht, das Gewicht der (für den Versand benötigten) Verpackung einer Ware. Das Bruttogewicht abzüglich der Tara ergibt das Nettogewicht, das meist der Preisberechnung und Verzollung zugrunde gelegt wird.“ (Brockhaus Enzyklopädie online s. v. Tara). Tara steht also laut Wörterbuch im Italienischen sowohl für Eigengewicht als auch für Verpackungsgewicht (ebenso wie peso dell'imballaggio; cf. Giacoma/Kolb 2019: 1197).

${ }^{31}$ Unter Füllgewicht versteht man laut Duden das „Gewicht einer Ware beim Einfüllen in ein Behältnis“ (cf. Duden online s. v. Füllgewicht. Die italienische Entsprechung (peso netto) kann unterschiedliche Bedeutungen haben (cf. Giacoma/Kolb 2019: 408). 
Handel] p. m netto; 2 (WASCHMASCHINE) capienza $\mathrm{f}$, carico m massimo" ([Z)]; Höchstg. - ,p. m massimo (consentito)“ ([Z] ; [ZS]); Ladeg. - „portata f (massima)“ ([Z] ; [ZS]); Lebendg. - ,p. m vivo“ ([Z]); Leerg. - ,p. m a vuoto“ ([Z]) $)^{32}$; Maximalg. - KE; Mindestg. - ,p. m minimo“ ([Z]); Nettog. - ,p. m netto“ ([Z]; [ZS]); Normalg. - „(genormt) p. m standard“ ([Z]); Reing. - ,p. m netto“ [Z]; Rohg. - ,p. m lordo“ ([Z]); Schlachtg. - KE; Trockeng ${ }^{33}$ - ,,p. m a secco“ ([Z)]/,p. secco“ ([T] $)^{34}$; Stückg. - KE; Verpackungsg. - ,tara f/p. m dell'imballaggio“ ([Z]); Volumeng./Volumg. - $p$. $m$ volumetrico $[\mathrm{Z}]$;

Naturwissenschaften (Mathematik, Physik, Chemie): Eigeng. - „phys (spezifisches Gewicht) p. m specifico“ ([Z]);

1b) Naturwissenschaften (Mathematik, Physik, Chemie): Atomg. - p. m atomico ([Z]); Gleichg.- KE (,equilibrio m chimico“ ([T])); Molekularg. - p. m molecolare ([Z]); Raumg. - KE;

32 Leergewicht hat laut DWDS folgende Bedeutung: „Gewicht des Fahrzeuges ohne Ladung“; „Synonymgruppe: Leergewicht [...] totes Gewicht“ (DWDS s. v. Leergewicht). Im einsprachigen deutschen Wörterbuch Duden online steht folgende Erklärung: „Gewicht eines Fahrzeugs in unbeladenem oder unbesetztem Zustand“; (cf. Duden online s. v. Leergewicht). Im Italienischen lautet die Entsprechung in Giacoma/Kolb: 'peso a vuoto'; 2019: 675) und in Treccani Vocabolario online: "peso a vuoto o peso morto" (,totes Gewicht"; ibd.), wobei nach Kontext bzw. Fachgebiet unterschieden wird; (cf. Treccani Vocabolario online s. v. peso ${ }^{2}$ ): „(A) In marina, p. morto, la portata lorda della nave costituita dal peso del carico, dei passeggeri, dell'equipaggio e delle dotazioni e provviste che la nave può trasportare in sicurezza e galleggiando“ bzw. im Flugverkehr: „(B) in aeronautica, $p$. a vuoto (o p. morto) di un aeromobile, il peso complessivo delle strutture, dei gruppi motopropulsori, dei serbatoi, dell'arredamento e delle installazioni varie, definite di volta in volta a seconda della natura e degli scopi dell'aeromobile“ (ibd.; Hervorhebungen G. B.). Die italienischen Bedeutungserklärungen dieser Bezeichnungen stimmen somit nicht mit jenen eines der beiden deutschen Wörterbücher überein. Mit dem Wort Leergewicht [Synonymgruppe:] „totes Gewicht“ ist nämlich „ein Fahrzeug ohne Ladung“ gemeint (cf. DWDS s. v. Leergewicht). Die Bedeutungserklärung im Treccani für $p$. morto lautet wörtlich ins Deutsche übersetzt: ,Tragfähigkeit des Schiffes, die sich aus dem Gewicht der Ladung, der Passagiere, der Besatzung und der Ausrüstung und der Vorräte zusammensetzt, die das Schiff sicher im schwimmenden Zustand befördern kann`. Die entsprechenden Einträge für Leergewicht und Deadweight (,totes Gewicht') im deutschen Nachschlagewerk geben Aufschluss: [Leergewicht:] „Gewicht des betriebsfertigen, nicht beladenen Kraftfahrzeuges einschließlich des gefüllten Kraftstoffbehälters und aller im Betrieb mitgeführten Ausrüstungsteile zuzüglich $75 \mathrm{~kg}$ als Fahrergewicht“" (Brockhaus Enzyklopädie online s. v. Leergewicht/Verkehrswesen); und die Erklärung für totes Gewicht lautet: „Deadweight ['dedwert; englisch ,totes Gewicht'] das, -(s)/-s, Abkürzung dw, die Masse der gesamten Zuladung (Ladefähigkeit, Tragfähigkeit) eines Schiffes, angegeben in tons deadweight (tdw; $1 \mathrm{tdw}=1016 \mathrm{~kg}$ ), auch in metrischen Tonnen, häufig in beiden. Das Deadweight umfasst Ladung, Besatzung, Brennstoff, Proviant und sonstige Verbrauchsstoffe“. (Brockhaus Enzyklopädie online s. v. Deadweight). Die Bedeutung von Deadweight-Tonnage (DWT) ist zudem das: „Maß für die Tragfähigkeit eines Handelsschiffes, [es] definiert die mögliche Nutzlast des Schiffes“" (Offizielles Stadtportal für Hamburg s. v. Deadweight Tonnage (DWT). In den Fachgebieten Verkehrswesen/Nautik sind die beiden Bezeichnungen Leergewicht / totes Gewicht somit nicht eindeutig synonym bzw. es existieren für deren Festlegung verschiedene mögliche Normen und Definitionen, die je nach Kontext berücksichtigt werden müssen.

33 „Gewicht der Ware in absoluter Trockenheit, errechnet aus dem Gewicht der aus einer Warenpartie gezogenen Muster nach dem Trocknungsprozess. Wichtig v. a. bei Waren, welche die Eigenschaft haben, Feuchtigkeit aus der Umgebung zu binden (hygroskopische Waren). Dient meist als Bezugsgröße für Inhaltsstoffe (z. B. Fettanteil pro Trockengewicht bei Käse)" (Gabler Wirtschaftslexikon online s. v. Trockengewicht).

${ }^{34}$ Für Trockengewicht finden sich zwei Entsprechungen im Italienischen: 1) peso a secco (cf. Giacoma/Kolb 2019: 1076); 2) ,[peso] secco [...] è il peso di una sostanza dopo eliminazione (per riscaldamento o con altri mezzi) dell'acqua che essa contiene“ (cf. Treccani Vocabolario online s. v. péso ${ }^{2}$; Hervorhebungen G. B.). 
2) 35 SSpr./Wirtschaft/Handel/Verkehrswesen/Mechanik: Bleigewicht (,Gardinen- Vorhanggewicht') - KE; (peso [T]); Bleigewicht (,Lot') [Seewesen] - KE; (peso di piombo (scandaglio) [T]) ${ }^{36}$; Gegeng. - contrappeso m [Z]; Münzg. - KE; (peso monetario [T]); Normalg. - “(genormt) 'p. m standard”" [Z]; Passierg. (Münzw.) - KE; (peso monetario [T]); Troyg.- KE; Zentnerg. - KE;

Naturwissenschaften (Mathematik, Physik, Chemie): Apothekerg. - KE; Eichg. - KE; Feing. ${ }^{37}$ - KE; Kilog. - KE;

Sport (Gewichtsklassen): Bantamg. - p. m gallo ([Z]); Federg. - p. m piuma ([Z]); Fliegeng. - p. m mosca ([Z]); Halbschwerg. - p. m medio-massimo ([Z]); Halbwelterg. (Boxen) - KE; Leichtg. - p. m leggero ([Z]; [ZS]); Leichtschwerg. - KE; Mittelg. - „1 (Gewichtsklasse) pesi m pl medi; 2 (Sportler) p. m medio“ ([Z]); [ZS]); Schwerg. - p . $\mathrm{m}$ massimo ([Z]); [ZS]); Welterg.- p. m welter;

3) Sspr./Theologie/Philosophie/Rechtswesen/u. a. (wenn nicht anders angegeben sind die it. Entspr. aus: Giacoma/Kolb 2019): Eigeng. - KE; Gegeng. ${ }^{38}$ - contrappeso m; Hauptg. - importanza f principale ([Z]); [ZS]); Schwerg. ${ }^{39}$ - massima importanza f; Überg. - KE; Ungleichg. - squilibrio ([Z]); [ZS]);

D) Komplexe Wortbildungen mit -gewicht- als Binnenglied und in Ableitungen ${ }^{40}$ :

1a) Sspr./Medizin: Gleichgewichtsempfinden/Gleichgewichtsgefühl - KE; gleich-gewichtig - equilibrato; Gleichgewichtslage - KE; Gleichgewichtsapparat (cf. Hammerschmid-Gollwitzer 1981: 154) - vestibolo [T]; Gleichgewichtsorgan - organo dell'equilibrio; Gleichgewichtssinn - senso dell'equilibrio ([Z]); [ZS]; Gleich-gewichtsstörung - alterazione f/disturbo dell'equilibrio; Gleichgewichtszustand - KE; schwergewichtig - essere pesantissimo, pesare molto; übergewichtig - (in) sovrappeso ([Z]); [ZS]; untergewichtig - sottopeso [Z]; [ZS];

1b) Naturwissenschaften: Gleichgewichtszustand - KE;

\footnotetext{
${ }^{35}$ Für die Gewichtsbezeichnungen in der Bedeutung (2) (,Körper mit einer bestimmten Schwere`) wurden in zweisprachigen Wörterbüchern (Giacoma/Kolb 2019; 2016) keine Einträge gefunden.

${ }^{36}$ Unter einem Bleigewicht ('Lot') (it. peso di piombo) versteht man im Seewesen eine „[mit Längenangaben markierte] Leine mit einem Bleigewicht zum Messen der Wassertiefe [...]“ (cf. Duden online s. v. Lot). Die Bedeutungserklärung der italienischen Entsprechung findet sich im Treccani Vocabolario online s. v. scandaglio).

${ }^{37}$ In der Bedeutung, Gewicht des in einer Münze enthaltenen Anteils an Edelmetall` (cf. Duden online s. v. Feingewicht).

38 Unter einem Gegengewicht versteht man ,[ein] Gewichtsstück zum Ausgleich einseitiger Belastungen, z. B. an Kranen (Standsicherheit), Aufzügen, oder zum Ausgleich von Fliehkräften, z. B. an Kurbelwellen von Verbrennungsmotoren“ (Brockhaus Enzyklopädie online s. v. Gegengewicht) bzw. standardsprachlich „Gewicht, das ein anderes Gewicht ausgleicht, aufhebt", wobei die Bezeichnung auch in übertragener Bedeutung verwendet wird, wie in ,seine Strenge bildet das notwendige Gegengewicht zu ihrer Nachgiebigkeit“ (Duden online s. v. Gegengewicht. Diese Bedeutungserklärung $(\mathrm{ER}=$ streng; $\mathrm{SIE}=$ nachgiebig $)$ entspricht allerdings eher Vorstellungen stereotyper Verhaltensweisen von „Männern“ und „Frauen“.

${ }^{39}$ Das Kompositum Schwergewicht kann auch in übertragener Bedeutung verwendet werden: „er/sie ist ein Schwergewicht [...] ein politisches Schwergewicht (jemand mit großem politischem Einfluss)“; (Duden online s. v. Schwergewicht).

${ }^{40}$ Die Übersetzungen (kursiv) stammen - wenn nicht anders angegeben (z. B. $[\mathrm{T}]=$ Treccani Vocabolario online - aus dem zweisprachigen deutsch-italienischen Wörterbuch Giacoma/Kolb (2019).
} 
2) Sport (it. Entspr. kursiv aus: Giacoma/Kolb 2019): Fliegengewichtler - KE ${ }^{41}$; Leichtgewichtler/-in; leichtgewichtig - KE; Mittelgewichtler/-in - KE; Schwergewichtler/in - KE; Schwergewichtsmeister/-in - campione/-ssa dei pesi massimi; Schwergewichtsmeisterschaft - campionato dei pesi massimi; Weltergewichtler - KE;

3) Sspr./Theologie/Philosophie/Rechtswesen/u. a.: gleichgewichtig - equilibrato ([Z]); Gleichgewichtszustand - KE; schwergewichtig - KE.

Für peso in der Gruppe A (einfaches Lexem) finden sich im einsprachigen Online-Wörterbuch Treccani weitere Einträge wie z. B. in der Bedeutung (1b) forza peso (,Gewichtskraft ${ }^{\star}$ ) oder in der Bedeutung (2) (,Gewichtsstücke'). Es handelt sich dabei um sogenannte Bleigewicht/e, die im Deutschen auch als Gardinen- und Vorhanggewichte oder Bleiband bekannt sind (cf. Treccani Vocabolario online s. v. péso ${ }^{2}$ ).

In der italienischsprachigen Literatur gibt es schließlich noch ein Beispiel mit peso in einer Bedeutung, die als Synonym für grado, condizione sociale (,Position/sozialer Status/Rang') gelten kann. Sie findet sich in Boccaccios Werk Il Decamerone ${ }^{42}$ und im folgenden Wortlaut „un giovinetto di non maggior peso di lei“ (cf. Treccani Vocabolario online s. v. peso ${ }^{2}$; i. d. deutschen Übersetzung: ,[...] eines jungen Menschen, an Stande nicht höher als sie“; Bocaccio 1783: 289; Hervorhebungen G. B.)

Für deutsche Komposita mit Gewicht als Erstglied (Gruppe B), mit und ohne Fugenelement, in den Bedeutungen (1a) und (1b) gibt es im Italienischen sowohl Entsprechungen im Singular als auch im Plural (peso, pesi), und ebenso in Kombination mit den Präpositionen di oder del (del/di peso; di pesi) bzw. ohne Präposition (Gewichtheben; it. sollevamento pesi). Vereinzelt sind auch Derivationen und abgeleitete Wortklassen möglich (wie z. B. in: Gewichtheber/-in - pesista; schwergewichtig - pesare molto; Ü/über-/U/untergewicht/-ig - sovrappeso/sottopeso; gewichten - soppesare). Auch andere deutsche Gewichtsbezeichnungen, für die es in den zweisprachigen Wörterbüchern keine Entsprechungen gibt, sind im einsprachigen Wörterbuch Treccani verzeichnet, wie z. B. im Fachgebiet der Physik oder Chemie (siehe Kapitel 3.2; cf. Treccani Vocabolario online s. v. péso ${ }^{2}$ ).

Für die insgesamt 63 Bezeichnungen im Deutschen mit Gewicht als Letztglied (siehe Gruppe C); (ink1. Zählung von Synonymen wie Volumeng./Volumg.) finden wir im Italienischen in den hier konsultierten ein- und zweisprachigen Wörterbüchern insgesamt 71 Entsprechungen bzw. Nullentsprechungen (KE). Die höhere Anzahl der italienischen Bezeichnungen ist einerseits bedingt durch vereinzelt mehrfache Entsprechungen für eine deutsche Bezeichnung (z. B. durch Angabe mehrerer Verwendungen oder Fachgebiete/Kontexte, für die es im Italienischen unterschiedliche Bezeichnungen gibt) und aufgrund z. T. unterschiedlicher Entsprechungen in einbzw. zweisprachigen Wörterbüchern (siehe Trockengewicht: in Giacoma/Kolb 2019/Treccani). Tabelle 3 stellt die italienischen Entsprechungen deutscher Gewichtsbezeichnungen der Gruppe C dar:

\footnotetext{
${ }^{41}$ Im Italienischen gibt es, anders als im Deutschen, keine Bezeichnungen für Sportler und Sportlerinnen der verschiedenen Körpergewichtsklassen (z. B. Fliegen-/Mittel-/Schwer-/Welter-/gewichtler/-in).

42 Es handelt sich dabei um das Kapitel: „Vierter Tag, Siebte Erzählung“ (Boccaccio 1866: 322).
} 


\begin{tabular}{|c|c|c|c|c|c|}
\hline Italienisch & (1a) & (1b) & (2) & (3) & Anzahl \\
\hline peso & & & $p$. & & 1 \\
\hline peso di & & & p. di piombo & & 1 \\
\hline peso del & dell'imballaggio & & & & 1 \\
\hline pesi & & & pesi $\mathrm{m} \mathrm{pl} \mathrm{medi}$ & & 1 \\
\hline peso $+\mathrm{N}$ & $\begin{array}{c}\text { forma } \\
\text { standard }\end{array}$ & & $\begin{array}{c}\text { gallo } \\
\text { mosca } \\
\text { piuma } \\
\text { welter }\end{array}$ & & 6 \\
\hline peso + Adj. & $\begin{array}{c}\text { anidro } \\
\text { complessivo/totale } \\
\text { corporeo } \\
\text { ideale (2) } \\
\text { massimo consen- } \\
\text { tito; } \\
\text { minimo } \\
\text { normale } \\
\text { secco } \\
\text { specifico } \\
\text { vivo } \\
\text { volumetrico }\end{array}$ & $\begin{array}{c}\text { atomico } \\
\text { molecolare }\end{array}$ & $\begin{array}{c}\text { leggero } \\
\text { massimo } \\
\text { medio } \\
\text { medio-massimo } \\
\text { monetario (2) }\end{array}$ & & 21 \\
\hline peso netto & $\begin{array}{l}\text { (Eigeng.) (Füllg.) } \\
\text { (Nettog.) (Reing.) }\end{array}$ & & & & 4 \\
\hline peso lordo & $\begin{array}{c}\text { (Bruttog.) } \\
\text { (Rohg.) }\end{array}$ & & & & 2 \\
\hline p. $+a+\mathrm{Adj}$. & $\begin{array}{l}\text { a secco } \\
\text { a vuoto }\end{array}$ & & & & 2 \\
\hline Deriv./Kompos. & $\begin{array}{c}\text { sottopeso } \\
\text { sovrappeso }\end{array}$ & & contrappeso & contrappeso & 4 \\
\hline andere & $\begin{array}{c}\text { capienza } \\
\text { carico massimo } \\
\text { equilibrio } \\
\text { portata (massima) } \\
\text { tara (2) }\end{array}$ & equilibrio & & $\begin{array}{c}\text { equilibrio } \\
\text { importanza } \\
\text { principale; } \\
\text { massima im- } \\
\text { portanza; } \\
\text { squilibrio }\end{array}$ & 11 \\
\hline $\mathrm{KE}$ & $\begin{array}{l}\text { (Abtropfg.) } \\
\text { (Frischg.) } \\
\text { (Maximalg.) } \\
\text { (Schlachtg.) } \\
\text { (Stückg.) }\end{array}$ & (Raumg.) & $\begin{array}{c}\text { (Apothekerg.) } \\
\text { (Bleig.); (Eichg.) } \\
\text { (Feing.) } \\
\text { (Halbwelterg.) } \\
\text { (Kilog.) } \\
\text { (Leichtschwerg.) } \\
\text { (Troyg.) } \\
\text { (Zentnerg.) }\end{array}$ & $\begin{array}{l}\text { (Eigeng.) } \\
\text { (Überg.) }\end{array}$ & 17 \\
\hline \multicolumn{5}{|c|}{ Anzahl italienischer Entsprechungen (inkl. KE) } & 71 \\
\hline
\end{tabular}

Tabelle 3: Italienische Entsprechungen deutscher Gewichtsbezeichnungen mit -gewicht als Letztglied (Gruppe C) 
Am häufigsten sind die Entsprechungen mit peso + Adj. $=(21)$ sowie mit peso $+\mathrm{N}$ (Nomen) (6). Sehr häufig findet man allerdings in den zweisprachigen Wörterbüchern keine Einträge $(\mathrm{KE}=(17))$. Italienische Entsprechungen ohne peso (andere $=(11)$ ) finden sich in der Gruppe $\mathrm{C}$ unter den Bedeutungen (1a), (1b) und (3). Vereinzelt gibt es für deutsche Gewichtsbezeichnungen im Italienischen polyseme Entsprechungen, die in mehreren Fachgebieten bzw. Zusammenhängen verwendet werden können (i. e. peso netto und peso lordo; peso ideale =, Idealgewicht'; ,Traumgewicht'). Die deutschen Bezeichnungen, für die es im Italienischen in zweisprachigen Wörterbüchern (Giacoma/Kolb 2019/2016) keine Entsprechungen gab, sind in Tabelle 3 in den Spalten 2 - 5 jeweils in Klammern gemäß den Bedeutungen von Gewicht ((1a), (1b), (2) und (3)) angegeben.

In der Gruppe D werden für gewichtig im zweisprachigen Wörterbuch zwei Bedeutungen im Italienischen unterschieden: „1) [gehoben] (bedeutend) [...] di un certo peso; importante; (MANN ${ }^{43}$, PERSÖNLICHKEIT) auch autorevole, di prestigio; 2) [scherzhaft] (korpulent) [...] grosso, corpacciuto“. Für Gewichtung wird das polyseme Nomen valutazione angegeben (cf. Giacoma/Kolb 2019: 455).

In Bezug auf die einzelnen Fachgebiete und die unterschiedlichen Bedeutungen von Gewicht sind somit keine „Eins-Zu-Eins-Entsprechungen“ (im Sinne von „Gewicht=peso“) festzustellen, sondern häufig Nichtentsprechungen im Sinne „Gewicht $\neq$ peso“ (siehe Tabelle 3).

\subsection{Wortverbindungen}

Unter „Wortverbindungen“ werden hier Wortverknüpfungen oder -kombinationen mit abgestufter Festigkeit (bis einschließlich Festigkeitsstufe 3) verstanden. Dies sind zumeist Verbindungen mit der Festigkeit 2, bei denen der Austausch einer Komponente möglich ist wie z. B. in Gewicht haben (avere peso/importanza; [Z]), an Gewicht verlieren (wörtlich im It.: ,perdere peso') oder etw anders/neu [gewichten] (,dare un peso diverso a qc, valutare qc diversamente $[\mathrm{Z}]$ ). In den genannten Fachgebieten werden außerdem einige Bezeichnungen mit Gewicht/peso verwendet, bei denen der Austausch einer Komponente nicht möglich ist, ohne die Gesamtbedeutung zu verändern. Diese Wortkombinationen besitzen somit eine Festigkeit 3 (z. B. im Italienischen sollevare pesi/*alzare pesi; deutsch ,Gewichte heben'). ${ }^{44}$

\subsubsection{Wortverbindungen mit „Gewicht“" in ein- und zweisprachigen Wörterbüchern}

In diesem Abschnitt werden Wortverbindungen mit Gewicht analog zur Darstellung in Kapitel 3.1 und 3.1.1 und gemäß den Bedeutungen (1a), (1b), (2) und (3) (siehe 2.1) eingeteilt. Dazu gehören unter (A) die Einträge ein- und zweisprachiger Wörterbücher. ${ }^{45}$ Belege zu Wortverbindungen mit Gewicht in Medientexten ${ }^{46}$ sind in Kapitel 3.2.2 dargestellt.

\footnotetext{
43 Dieser Bedeutungszusatz steht nur in Giacoma/Kolb 2019, nicht in Giacoma/Kolb 2016.

44 Der Begriff „Festigkeit“ wird von Wanzeck nach unterschiedlichen Stufen (von 1 bis 3) definiert (2010: 103109). Die unter (A) angegebenen italienischen Übersetzungen stammen aus den Wörterbüchern Giacoma/Kolb (2016, [ZS]) sowie Giacoma/Kolb (2019, [Z]).

45 Die italienischen Entsprechungen (kursiv) stammen aus aus den zweisprachigen Wörterbüchern Giacoma/Kolb (2019, [Z]; (2016, [ZS]) und aus dem einsprachigen Wörterbuch Treccani Vocabolario online s. v. péso ${ }^{2}$ [T].

46 Texte deutscher Medien (ARD; ZDF; Deutschlandfunk (Dlf)) aus dem Zeitraum 2017-2020.
} 
(A)

1a) Sspr./Medizin: ein G. haben von x kg - pesare x $\mathrm{kg}$ ([Z]); großes/geringes G. haben avere $[\ldots]$ molto/(un) gran)/poco p. [Z];

Handel/Verkehrswesen/Mechanik: zulässiges Gewicht - peso consentito [Z]; [ZS]; das Gewicht (nicht) überschreiten - (non) passare il peso [Z]; das Gewicht (nicht) unterschreiten - il peso non deve essere inferiore $a$; nach Gewicht kaufen/verkaufen comprare/vendere a peso [Z]; [ZS]; [T];

Sport: , $<$ meist pl $>$-e heben - sollevare pesi $([\mathrm{ZS}])$;

Naturwissenschaften: spezifisches Gewicht - ,(nur sing $>$ ) il peso specifico“ ([ZS]);

1b) KE;

2) ${ }^{47}$ Handel/Verkehrswesen/Mechanik: große, kleine Gewichte - KE; die Gewichte der Pendeluhr ${ }^{48}$ - KE; die Gewichte müssen geeicht sein - KE; mehrere Gewichte auf die Waage legen - KE; bleierne Gewichte (,aus Blei') (cf. DWDS s. v. bleiern) - pesi di piombo [T].

Sport: ein Gewicht stemmen/reißen/drücken - KE; Gewichte heben - sollevare pesi [Z];

3) Sspr./Theologie/Philosophie/Rechtswesen/u. a.: großes/geringes Gewicht haben - avere molta/poca importanza [Z]; ein Bleigewicht stemmen (cf. DWDS s. v. Bleigewicht) - KE; bleierne Gewichte (,schwer lastend') (ibd.) - KE.

Im einsprachigen Wörterbuch Treccani (cf. Treccani Vocabolario online s. v. peso ${ }^{2}$ ) sind außerdem einige zwei- und mehrteilige Gewichtsbezeichnungen mit den Bedeutungen (1a) und (1b) aufgeführt, die Fachgebieten zugeordnet werden können. Dazu gehören im Fachgebiet Wirtschaft/Handel mit Bezug auf die Bedeutung (1a) die Bezeichnung $p$. d'origine (o all'origine) (siehe dazu Kapitel 2.2.2) sowie im Sport die Verbindung recinto del peso ${ }^{49}$, was im Deutschen einem ,Waagegebäude' entspricht. Dazu die folgende Erklärung: „Das Waagegebäude trägt seinen Namen, weil hier die Jockeys vor und nach den Rennen gewogen werden"; cf. Hamburger Renn-Club e.V.). Für (1b) gibt es außerdem einige, meist zweigliedrige, Wortverbindungen in naturwissenschaftlichen Fachgebieten.

In der Physik: p. specifico relativo (im Deutschen: ,relative Dichte'; auch ,spezifische Dichte $\left.{ }^{60}\right)$.

\footnotetext{
${ }^{47}$ Die Beispiele von Wortverbindungen mit Gewicht in der Bedeutung (2) stammen aus Duden online (cf. Duden online s. v. Gewicht), dem einsprachigen Wörterbuch Treccani (Treccani Vocabolario online) und dem zweisprachigen Wörterbuch Giacoma/Kolb (2019).

${ }^{48}$ Für diese Kombination gibt es in den zweisprachigen Wörterbüchern keine Einträge, sondern nur das folgende Beispiel im einsprachigen Wörterbuch Treccani: ,in alcuni orologi (orologi a peso), la massa materiale che, per la forza di gravità, agisce come organo motore" (Treccani Vocabolario online s. v. péso ${ }^{2}$; Hervorhebungen G. B.; ins Deutsche übersetzt ,bei einigen Uhren (Gewichtsuhren) die materielle Masse, die durch die Schwerkraft als Triebkraft wirkt'). Bei den sogenannten Gewichtsuhren handelt es sich um eine der beiden Hauptarten von Uhren. Die zweite Hauptart sind Federuhren.

${ }^{49}$ Nach der Bedeutungserklärung im einsprachigen Wörterbuch Treccani: „l'ambiente degli ippodromi in cui si pesano i fantini prima e dopo la gara, detto anche, con termine fr. pesage" (Treccani Vocabolario online s. v. péso $o^{2}$.

50 Die Definition lautet im Deutschen wie folgt: „,das Verhältnis der Dichte [...] eines Stoffes zur Dichte [...] eines Normzustandes dieses Stoffes“; (siehe Kapitel 3.2.1).
} 
In der Chemie: $p$. equivalente (o di combinazione). Im Deutschen lautet die Entprechung „,Äquivalentmasse“ [,früher Äquivalentgewicht"]“ (Brockhaus Enzyklopädie online s. v. Äquivalentmasse); p. formula (relativo) di un composto (deutsch: „Molmasse einer Verbindung'; früher: Formelgewicht"; cf. Brockhaus Enzyklopädie online s. v. Molare Masse).

Sowie in der Bodenkunde: ,p. specifico apparente [oder] p. di volume (il peso dell'unità di volume della roccia)" (cf. Treccani op. cit.). Im Deutschen lautet die entsprechende Bezeichnung ,Lagerungsdichte , auch Raumgewicht oder Volumengewicht genannt“ (cf. Brockhaus Enzyklopädie online s. v. Lagerungsdichte(Bodenkunde).

Auch im Fachgebiet Rechtswesen („Erbrecht“) führt das einsprachige Wörterbuch für das Italienische ein Beispiel mit peso an (Treccani op. cit.). Es handelt sich um die Wortverbindung p. ereditarî (wörtlich: ,geerbte Gewichte'). Im Deutschen spricht man dabei in fachsprachlichen Zusammenhängen von Nachlassverbindlichkeiten (cf. Brockhaus Enzyklopädie online s. v. Nachlassverbindlichkeiten und ibd. s. v. Erbrecht).

\subsubsection{Wortverbindungen mit Gewicht in Medientexten}

Zusätzlich zu den genannten Einträgen in Wörterbüchern und Nachschlagewerken sind in Medientexten folgende freie Kombinationen (Festigkeit 1) mit Gewicht in den Bedeutungen (1a) geläufig (siehe 2.1), d. h. vor allem in Verweisen auf Körpergewicht:

(1a) (wieder) Gewicht aufbauen (Dlf, 26.05.2017); im Gewicht schwanken (Dlf, 11.11.2018); (an) Gewicht verlieren (ZDF, 19.02.2018; ARD, 22.01.2019); auf das (eigene)/sein/ihr/Gewicht achten (ZDF, 19.06.2018); sein Gewicht halten (ZDF, 15.09.2019); bei Idealgewicht (ARD, 30.05.2019); ein Gewicht von [...] kg haben (ZDF, 02.09.2019); mit einem Gewicht von [...] nur den/einen Bruchteil des Gewichts + GA ${ }^{51}$ + auf die Waage bringen (ZDF, 02.09.2019; Hervorhebungen G. B.).

Dazu der folgende Nachrichtentext:

Pandas bringen eines oder zwei, selten sogar drei Jungtiere auf einmal zur Welt. Sie werden nach rund vier bis sechs Monaten Tragzeit nur spärlich behaart und blind geboren. Mit einem Gewicht von meist nur 90 bis 130 Gramm bringt der Nachwuchs nur einen Bruchteil des Gewichts seiner Mutter auf die Waage. Laut Zoo [...].

(ZDF, 02.09.2019; Hervorhebungen G. B.)

(1b) mit einem Gewicht von [...] Tonnen/kg (ARD, 05.07.2019); von dem/vom Gewicht her (,das Gewicht betreffend'; (ARD, 27.08.2018); das Gewicht + GA (nach)messen (...); unter dem Gewicht [+ GA] einstürzen [...]; das Gewicht [+ GA] (nicht) tragen/halten (können) (ARD, 14.12.2020; Hervorhebungen G. B);

Beispiele von Wortverbindungen mit Gewicht im Kontext (von dem/vom Gewicht her; das Gewicht nicht halten können) sind z. B. folgende:

Die schwierigste Aufgabe [...] war es, eine [...] zu erfinden, die [...] von der Größe und dem

Gewicht her so klein und leicht wie möglich war.

(ARD, 27.08.2019; Hervorhebungen G. B.)

${ }^{51} \mathrm{GA}=$ Genitivattribut 
Und Plastik kann das Gewicht des Schnees nicht halten. Deshalb können wir uns nicht vorstellen, dass wir in der Winter- und Schneesaison hier leben können.

(ARD, 14.12.2020; Hervorhebungen G. B.)

In Medientexten wird der Gebrauch von Wortverbindungen mit Gewicht in den Bedeutungen (1a) im Singular bestätigt. Nach Gewicht folgt häufig ein Genitivattribut (GA), wenn man sich auf das ,Gewicht' einer Person (,Körpergewicht') oder einer Materie (i. e. eines Stoffes oder einer Substanz) bezieht. Dabei kann auch ein Präpositionalobjekt im Dativ (nach von) verwendet werden, z. B. wenn es sich um eine unbestimmte oder bestimmte Mengenangabe (wie z. B. Stahl; [...] kg) handelt.

In Medientexten finden sich dagegen nur wenige Beispiele von Wortverbindungen mit Gewicht (meist im Plural) in der Bedeutung (2) wie z. B. Gewichte eichen (Dlf, 16.11.2018). Geläufig sind u. a. zweigliedrige Kombinationen mit Gewicht im Plural und einem Verb im Zusammenhang mit dem Sport (i. e. den Bereichen Agonistik und sportliches Training allgemein) sowie mit dem Messwesen, wie z. B. in:

(2) Gewichte heben (wollen): „Die Leute wollen [...] Gewichte heben“ (ARD, 17.08.2018); Gewichte stemmen: „Gewichte stemmen im Fitness-Studio ist nicht ganz risikofrei” (Dlf, 21.06.2019)

Wortverbindungen mit Gewicht gemäß Bedeutung (3) (siehe Abschnitt 2.1) sind dagegen auch in zweisprachigen Worterbüchern aufgeführt (cf. Giacoma/Kolb 2016 [ZS]; 2019 [Z]):

Gewicht haben \{ARGUMENT\}, avere peso/importanza [ZS]; großes/geringes [Gewicht] haben avere $[\ldots]$ molto/(un) gran $[\ldots] /$ (poco) peso, avere molta/poca importanza [Z]; seine Stimme hat entscheidendes Gewicht - il suo voto ha un peso decisivo/determinante [Z].

(Giacoma/Kolb 2016 [ZS]; 2019 [Z])

In Texten deutscher Medien finden sich in der Bedeutung (3) von Gewicht zahlreiche Wortverbindungen, darunter vereinzelt auch mit Gewicht im Plural:

(3) mit + Adj (Komparativ) z. B. in: „,mit größer-/stärker-/wachsendem Gewicht“; (ARD, 26.05.2019); (überhaupt) kein/[...]politisches) Gewicht haben (z. B. in: „die Europäer [haben] überhaupt kein sicherheitspolitisches und nur ein sehr begrenztes außenpolitisches

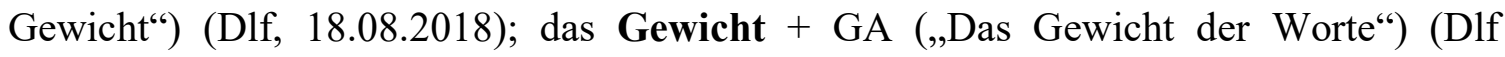
17.02.2020); (mehr) Gewicht bekommen/erhalten/fordern [...]; etwas stärker gewichten; das nötige [...] Gewicht fehlen (,dafür fehlt ihm [...] das nötige militärische Gewicht“) (Dlf 08.09.2018); genug Gewicht auf die Waage bringen (Dlf, 21.07.2017); von Gewicht (sein) (Dlf Kultur, 18.11.2018); die Gewichte in [...] verschieben sich (,die Allianzen/Einflussnahme/Prioritäten verändern/t sich; betreffen/betrifft andere Gebiete') (ARD, 12.11.2018; sämtliche Hervorhebungen G. B.).

Die geläufigsten lexikalischen Ergänzungen von Gewicht in der Bedeutung (3) in Medientexten sind einerseits Verben (z. B. haben/sein, bekommen/erhalten, geben und fordern), Adjektive (auch im Komparativ), Partizipien, Adverbien oder Ortsangaben, mit oder ohne Artikel wie z. B. ein hohes Gewicht; mehr/großes/geringes/politisches/starkes/stärkeres + politisches/ökonomisches/wachsendes/wirtschaftliches Gewicht; meist in Verbindung mit den oben genannten Verben. In Wortverbindungen wird auch manchmal das Nomen Waage verwendet (z. B. in: 
(ein) (+ Adverb/Adjektiv) Gewicht auf die Waage bringen) im Sinne von ,bedeutsam sein/Bedeutung haben'.

Die Wortverbindung das/sein/ihr Gewicht halten kann sich in Bedeutung (1a) (,Schwere eines Körpers', siehe Abschnitt 2.1) auf das Körpergewicht einer Person beziehen. Die Verbindungen (an) Gewicht gewinnen/zunehmen bzw. das Gegenstück dazu (an) Gewicht verlieren werden sowohl im Zusammenhang mit Körpergewicht als auch in der Bedeutung (3) von Gewicht verwendet. Der Gebrauch der Präposition an (vor Gewicht) ist dabei in der übertragenen Bedeutung (3) etwas häufiger zu beobachten, als in Bedeutung (1a). Dazu die folgenden Beispiele: „Ich habe fünf Monate vor dem Drehstart im Oktober 2014 aufgehört, Sport zu treiben, denn ich musste Gewicht [...] verlieren“ (ARD, 22.01.2019) sowie: „Gerade beim Namen und der Postanschrift geben aber viele YouTube-Nutzer Fantasiedaten an. Damit können die Urheber naturgemäß nicht viel anfangen. Darum gewinnen die übrigen Daten an Gewicht" (ARD, 18.02.2019).

\subsection{Redewendungen mit Gewicht/peso in ein- und zweisprachigen Wörterbüchern}

Unter (A) werden Redewendungen mit Gewicht als Komponente (einschl. Derivationen) aufgeführt, die im zweisprachigen Wörterbuch (Giacoma/Kolb 2019 [Z]) verzeichnet sind:

(A) ins Gewicht fallen (,von großer, ausschlaggebender Bedeutung sein') ${ }^{52}$ - avere molto peso/importanza; essere importante; auf etwas Gewicht legen (, es für wichtig erklären'53) - dare peso/importanza a qc; etwas Gewicht beimessen - dare peso/importanza a qc; sein ganzes [Gewicht] für jdn/etw in die Waagschale werfen - usarelfar valere tutta la proria influenza/tutto il proprio prestigio per qu/qc; etwas anders/neu gewichten - dare un peso diverso a qc, valutare qc diversamente.

Diese Redewendungen (z. B. ins Gewicht fallen; Gewicht legen auf; sein/ihr Gewicht in die Waagschale werfen) werden in Medientexten häufig durch linke Nachbarn wie z. B. Adjektive, Adverbien oder Negationen modifiziert. Die übertragene Bedeutung mutiert durch diese Ergänzungen nicht grundlegend, sie kann allerdings entsprechend eingeschränkt oder präzisiert werden. Auch die Verwendung unterschiedlicher Tempora oder Modi (i. e. Konjunktiv II) führt zu Bedeutungsnuancen.

Im einsprachigen italienischen Wörterbuch sind mit der Komponente peso (abgekürzt: p.) folgende Redewendungen verzeichnet (siehe Beispiele (1) - (5)) (cf. Treccani Vocabolario online s. v. péso ${ }^{2}$ ). Sie werden der Reihenfolge nach jeweils mit ihren wörtlichen deutschen Übersetzungen und ihren Entsprechungen in ein- und zweisprachigen Nachschlagewerken angegeben ([D]: Duden (2020a; 2019); Duden online); [Z]: Giacoma/Kolb 2019: 2100):

\footnotetext{
52 Die „konkrete, sinnliche Bedeutung“ der Redewendung ,,ins Gewicht fallen“ tritt laut Duden „hier nicht klar hervor, und der konkrete Bezug wird vielleicht klarer, wenn man Gewicht als „Waagschale“ oder „Wiegegerät“ deutet, das mit dem „fallenden Gewicht“ dann den Ausschlag gibt.“ (Duden 2014: 29).

53 Zur Bedeutungserklärung: 1) „Gewicht auf etwas legen = es für wichtig erklären; [deutsche Redewendung. Der Ausdruck stammt ursprünglich aus der Kaufmannssprache] „früherer Zeiten“““. (Cf. Mackensen 1981: 324); 2) „Das sprachliche Bild bezieht sich auf die Waagschale, auf die man noch ein Gewicht legt, damit die Zunge nach ihrer Seite ausschlägt“" (Duden 2014: 276).
} 
(1) sentire un p. sullo stomaco (per aver mangiato cibi di difficile digestione), [wörtlich: ,ein Gewicht auf dem Magen spüren' (,nach dem Verzehr schwerverdaulicher Speisen')]; [Z]: ,ein Schweregefühl im Magen haben'; deutsche Redewendung [D]: jdm [schwer wie Blei] im Magen liegen (cf. Duden (2020a: 498); [Z]: avere un peso sullo stomaco (sensazione di pesantezza); ,etw (schwer) im/[auf dem] Magen liegen haben“;

(2) togliersi un p., un bel p. dallo stomaco (liberarsi da una preoccupazione, da un problema angosciante), [wörtlich: ,(sich) ein Gewicht/ein schönes Gewicht vom Magen entfernen/(weg)nehmen', (,sich von einer Sorge, von einem beängstigenden Problem befreien')]; [Z]: togliersi una peso dallo stomaco/dal cuore: ,mir ist ein Stein vom Herzen gefallen'; deutsche Redewendung [D]: jdm fällt ein Stein/(scherzh.:) ein Steinbruch vom Herzen (cf. ibd.: 725);

(3) sentire il p. della vita, degli anni, del lavoro [wörtlich: ,das Gewicht des Lebens, der Jahre, der Arbeit spüren']; [Z]: die Bürde des Alters; ${ }^{54}$ geläufige Wortverbindungen im Deutschen: die Bürde des Lebens/des Alters/der Jahre; 55 das Gewicht der Arbeit.

(4) essere un p. per qu/non voler essere un p. a nessuno (condizione, situazione, evento che grava su una persona causandole preoccupazioni, disagio, tormento, o implicando serie responsabilità); [wörtlich: ,ein Gewicht für jmdn sein/kein Gewicht für jmdn sein wollen'; (,Zustand/Umstand, Situation oder Ereignis, der/die/das eine Person belastet und ihr (große) Sorgen, Kummer bereitet oder eine große Verantwortung bedeutet')]; [Z]: deutsche Redewendung [D]: jmdm zur Last fallen; niemandem zur Last fallen wollen;

(5) avere, adottare, usare due pesi e due misure (essere ingiusto, parziale nel proprio giudizio, adoperare criterî diversi a seconda dell'oggetto o delle persone ai quali si applicano); [wörtlich: ,zwei Gewichte und zwei Maße haben, anwenden, verwenden` (,ungerecht sein, parteiisch in seinem Urteil sein, je nach Sache oder Person unterschiedliche Urteilskriterien anwenden')]; [Z] und [D]: mit zweierlei Maß messen.

Die o. a. italienischen Redewendungen (1) - (5) kennt man in ähnlicher Form und Bedeutung auch im Deutschen. Jedoch werden neben Gewicht auch andere Komponenten verwendet, wie z. B. in (1) sentire un peso (wörtlich: ,ein Gewicht spüren'); deutsch: schwer im Magen liegen oder in (2) togliersi un peso dallo stomaco (wörtlich: ,sich ein Gewicht vom Magen nehmen/entfernen'); deutsch: jdm fällt ein Stein vom Herzen; ebenso in (3) und (4) sentire un peso bzw. essere/non (voler) essere un peso per qu; deutsch: Bürde [...] tragen bzw. jdm/niemandem zur Last fallen (wollen) und schließlich in (5) avere, adottare, usare due pesi e due misure; deutsch nur mit der Komponente Maß i. e. ohne Gewicht.

Eine tabellarische Darstellung stellt diese Unterschiede zwischen den italienischen und deutschen Redewendungen dar (Hervorhebungen G. B.; siehe auch Tabelle 4):

1) sentire un peso sullo stomaco $=, \mathrm{jdm}$ [schwer wie Blei] im Magen liegen';

2) togliersi un peso, un bel peso dallo stomaco = ,jdm fällt ein Stein/Steinbruch vom Herzen';

\footnotetext{
${ }^{54}$ Die italienische Entsprechung der deutschen Wortverbindung findet man bei dem Eintrag Bürde (,il peso della vecchiaia, il gravame degli anni'); (cf. Giacoma/Kolb 2019: 265).

55 Wortverbindungen mit Bürde in der Bedeutung, seelische Last` (cf. DWDS s. v. Bürde).
} 
3) sentire il peso della vita, degli anni, del lavoro = , die Bürde des Lebens/des Alters; das Gewicht der Arbeit ${ }^{56}$ tragen"; $^{\text {; }}$

4) essere un peso per qu/non voler essere un peso a nessuno = ,jmdm zur Last fallen; niemandem zur Last fallen wollen';

5) avere, adottare, usare due pesi e due misure =, mit zweierlei Maß messen'.

\begin{tabular}{|c|c|c|}
\hline $\begin{array}{l}\text { IT. REDEWENDUNGEN MIT DER } \\
\text { KOMPONENTE PESO, s. m. }\end{array}$ & wörtliche deutsche BEDEUTUNG & $\begin{array}{l}\text { DEUTSCHE REDEWEN- } \\
\text { DUNGEN UND WORTVER- } \\
\text { BINDUNGEN } \\
\end{array}$ \\
\hline (C) sentire un peso sullo stomaco & $\begin{array}{l}\text {,ein Gewicht auf dem Magen } \\
\text { spüren' }\end{array}$ & $\begin{array}{c}\text { schwer }[\text { wie Blei }] \text { im Magen } \\
\text { liegen }\end{array}$ \\
\hline $\begin{array}{l}\text { (D) togliersi un peso/un bel peso } \\
\text { dallo stomaco }\end{array}$ & $\begin{array}{l}\text {,sich ein Gewicht/ein schönes } \\
\text { Gewicht vom Magen nehmen' }\end{array}$ & $\begin{array}{l}\text { jdm fällt ein Stein vom Her- } \\
\text { zen }\end{array}$ \\
\hline $\begin{array}{c}\text { (E) sentire il peso della vita, degli } \\
\text { anni, del lavoro }\end{array}$ & $\begin{array}{c}\text {,das Gewicht des Lebens, der Jahre, } \\
\text { der Arbeit spüren“ }\end{array}$ & $\begin{array}{c}\text { die Bürde des Lebens/des Al- } \\
\text { ters/der Jahre tragen; das Ge- } \\
\text { wicht der Arbeit }\end{array}$ \\
\hline (F) essere un peso per qu & ,ein Gewicht für jmdn Sein` & jmdm zur Last fallen \\
\hline $\begin{array}{c}\text { (F) non voler essere un peso a nes- } \\
\text { suno }\end{array}$ & $\begin{array}{l}\text {,für niemanden ein Gewicht sein } \\
\text { wollen` }\end{array}$ & $\begin{array}{l}\text { niemandem zur Last fallen } \\
\text { wollen }\end{array}$ \\
\hline $\begin{array}{c}\text { (G) avere, adottare, usare due pesi e } \\
\text { due misure }\end{array}$ & $\begin{array}{c}\text {,zwei [unterschiedliche] Gewichte } \\
\text { und zwei [unterschiedliche] Maße } \\
\text { haben/anwenden/gebrauchen` }\end{array}$ & mit zweierlei Maß messen \\
\hline
\end{tabular}

Tabelle 4: Redewendungen mit der Komponente peso und ihre Entsprechungen im Deutschen

\section{Deutsch-italienische Übersetzungsbeispiele}

Internetportale mit deutsch-italienischen Übersetzungsbeispielen sind eine zusätzliche Quelle bei der Suche nach italienischen Bezeichnungen. Im folgenden Abschnitt werden Beispiele von Lexemen und Wortverbindungen dargestellt, die in einschlägigen Wörterbüchern nicht aufgeführt sind.

\subsection{Deutsche Belege mit Gewicht und italienischen Übersetzungen}

Deutsch-italienische Belege mit Gewicht in den Bedeutungen (1a) und (1b) (siehe 2.1)57; Hervorhebungen G. B.):

(I) Beispiele mit Gewicht $=$ peso

dt.: Das Gewicht der Stichprobe darf $250 \mathrm{~g}$ nicht unterschreiten.

ital: Il peso del campione non deve essere inferiore a 250 grammi.

\footnotetext{
56 „Das Gewicht der Arbeit“ ist auch der deutsche Titel eines Gemäldes von Paul Hannon („,The Weight of Labour“, 2016). Da Arbeit im Deutschen auch ,Schriftstück“ bedeuten kann, wird die Wortverbindung im universitären Kontext verwendet im Sinne von „Gewichtung bzw. Feststellung von Daten zur Beurteilung einer schriftlichen Arbeit“" (cf. Prüfungsordnung für den Bachelorstudiengang Mathematik).

57 Die deutsch-italienischen Übersetzungen stammen vom Reverso Übersetzungsportal ('context.reverso') unter dem Stichwort Gewicht.
} 
dt.: Eine Zeitung kann achtmal gefaltet eine Tonne Gewicht aushalten. ital: I giornali piegati otto volte possono sopportare il peso di una tonnellata.

(II) Beispiele mit Gewicht = massa

dt.: Liegt das Gewicht des Anhängers in unbeladenem Zustand über 75 \% des Höchstgewichts, sind nur die Grenzen für den „,beladenen“ Zustand anzuwenden.

ital: Se la massa a vuoto del rimorchio è superiore al $75 \%$ della massa massima, i limit devono essere applicati unicamente per la condizione "veicolo carico".

dt.: Das Gewicht des unlöslichen Bestandteils wird als Prozentsatz des Gesamtgewichts der im Gemisch enthaltenen Fasern ausgedrückt.

ital: Si esprime la massa del componente insolubile come percentuale della massa totale delle fibre presenti nella mischia.

Die o. a. Beispiele zeigen, dass man im Italienischen unterscheidet, ob Gewicht in der Bedeutung einer ,gravitationsunabhängigen Masse eines Körpers' (it. massa) oder im Sinne von ,Gewichtskraft' eines Gegenstands (it. peso/forza peso) verwendet wird (siehe 2.1).

\subsection{Belege mit Gewicht im Sinne von ,Bedeutung, Wert, Wichtigkeit' und italienischen Übersetzungen}

Es gibt eine ganze Reihe deutscher Texte, in denen Gewicht in der Bedeutung (3) verwendet wird (siehe 2.1). Laut Wörterbücher heißt die Entsprechung im Italienischen importanza. Werden mit Gewicht auch Attribute oder Adverbien verwendet, vergrößert sich die Palette der italienischen Entsprechungen der Übertsetzungsportale, und wir finden dort eine Reihe italienischer Nomina wie z. B. accento/attenzione/enfasi/importanza/rilevanza, die in den folgenden Beispielen mit Gewicht als Lexem, sowie als Komponente in Wortverbindungen und Redewendungen exemplarisch dargestellt sind. Gewicht nach Bedeutung (3) (,Bedeutung, Wert') mit italienischen Entsprechungen (siehe (a) - (d); Hervorhebungen G. B.):

(a) Gewicht-importanza

dt.: Das Gewicht des Europäischen Parlaments und die Rolle der nationalen Parlamente nehmen weiter zu.

ital: L'importanza del Parlamento europeo e il ruolo dei parlamenti nazionali saranno rafforzati.

(b) entscheidendes Gewicht zukommen - avere una rilevanza notevole

dt.: Das häufige Überfluten der Grundstücke hat zu ihrer zunehmenden Versalzung geführt, wodurch die Ernten vernichtet werden und die künftige Eignung zur landwirtschaftlichen Nutzung des Bodens dieser Gemeinde, in der der Landwirtschaft entscheidendes Gewicht zukommt, gefährdet ist.

ital: Le frequenti inondazioni hanno provocato la progressiva salinizzazione dei terreni, distruggendo le colture e minacciando la possibilità futura di utilizzare per scopi agricoli $\mathrm{i}$ terreni di questa località, nella quale l'agricoltura ha una rilevanza notevole.

(c) besonderes Gewicht beimessen/haben - rivolgere una particolare attenzione a/prestare particolare attenzione a

dt.: Bei dieser Thematik ist den Biokraftstoffen und der Notwendigkeit von Vorschriften und Standards für ihre Vermarktung besonderes Gewicht beizumessen. 
ital: In tale contesto, è opportuno rivolgere una particolare attenzione ai biocombustibili e alla necessità di definire regole e norme per la loro commercializzazione.

dt.: Besonderes Gewicht muss den Themen politische Legitimität, demokratische Kontrolle und Transparenz bei den Entscheidungen beigemessen werden.

ital: Bisogna prestare particolare attenzione a questioni quali la legittimazione politica, il controllo democratico e la trasparenza nel processo decisionale.

dt.: Besonderes Gewicht wird auch die Einbeziehung von Partnern aus den neuen Mitgliedstaaten und den Bewerberländern haben.

ital: Un'attenzione particolare sarà prestata al coinvolgimento dei nuovi Stati membri e dei paesi candidati.

(d) größeres Gewicht beimessen - attribuire maggiore importanza a

dt.: Es scheint nötig zu sein, diesem Thema ein größeres Gewicht beizumessen.

ital: Risulta necessario attribuire maggiore importanza a questo problema.

In den folgenden Textausschnitten sind Beispiele der Redewendung Gewicht legen auf zusammen mit italienischen Übersetzungen dargestellt. Dabei wird zwischen einer einfachen Form sowie den Modifikationen der Redewendung (i. e. Ergänzungen mit Adverbien oder Adjektiven) unterschieden. Während man für die ,einfache' deutsche Version im Italienischen häufig porre l'accento su qc findet, werden Modifikationen z. B. Ergänzungen (Adverbien o. a.) wie auf etwas mehr Gewicht legen im Italienischen ebenfalls mit verstärkenden Zusätzen übersetzt wie mit porre maggiore enfasi su qc oder porre un'enfasi particolare su qc. Dazu die folgenden Beispiele (e) - (g); Hervorhebungen G. B.):

(e) Gewicht legen auf etwas - porre l'accento su qc

dt.: Bei dem Änderungsantrag wird das Gewicht auf die Sicherheit und Gesundheit der Anwender gelegt.

ital: L'emendamento pone l'accento sulla salute e la sicurezza dell'utilizzatore.

dt.: Das Gewicht wurde auf die Ausarbeitung von Normen für die wesentlichen Bereiche des Umweltschutzes gelegt.

ital: L'accento veniva posto sull'elaborazione di norme interessanti i principali aspetti della politica ambientale.

(f) auf etwas mehr Gewicht legen-porre maggiore enfasi su qc

dt.: Auf die Verwertung von organischen Abfällen, gerade in dieser weitgehend landwirtschaftlich geprägten Region, muss mehr Gewicht gelegt werden.

ital: Maggiore enfasi deve essere posta sul recupero dei rifiuti organici, in modo particolare in questa regione a vocazione prevalentemente agricola.

(g) besonders viel Gewicht legen auf etwas - porre un'enfasi particolare su qc

dt.: Wir sollten tatsächlich besonders viel Gewicht auf das lebenslange Lernen legen, den sogenannten Lifelong learning process.

ital: In effetti dobbiamo porre un'enfasi particolare sull'istruzione e sulla formazione professionale continua, o Lifelong learning process.

\subsection{Auswertung}

In den Fachsprachen der Naturwissenschaften, der Mechanik und im Sport gibt es bei der Verwendung von Gewicht/peso im Deutschen und im Italienischen lexikalische 
Übereinstimmungen. Dies betrifft den Gebrauch in den Bedeutungen (1a) und (2) einschließlich Fachtermini des metrischen Gewichtssystems sowie Gewichtsklassen im Sport. Für Gewicht im Singular verwendet man im Italienischen allerdings auch gelegentlich peso im Plural (pesi), und für Komposita und Derivationen mit Gewicht als Erst- oder Letztglied finden sich als Entsprechungen im Italienischen ebenfalls Ableitungen/Zusammensetzungen, oder es gibt dafür in den zweisprachigen Wörterbüchern keine Einträge (siehe Abschnitt 3.1.1; Tabelle 3).

Komposita mit Gewicht- als Erstglied (siehe 3.1.1 Gruppe B) werden im Italienischen gemäß den geltenden Sprachregeln je nach Kontext entweder im Singular oder im Plural bzw. mit den Präpositionen wie di oder del (peso) verwendet. Häufig sind diese Entsprechungen mehrteilig und bestehen aus ,N (Nomen) + di peso' (z. B. in: aumento/categoria/perdita/problema/unità di peso) oder aus , $\mathrm{N}+$ del peso'. Dafür konnten Entsprechungen im Handel/in der Wirtschaft sowie gelegentlich auch im Sport mit (controllo/indicazione del peso; spostamento del peso) ermittelt werden.

Unter den italienischen Entsprechungen mit -gewicht als Letztglied (siehe 3.1.1 Gruppe C) gibt es sehr häufig zweigliedrige Verbindungen wie ,peso + Adjektiv‘ (wie in: peso atomico/corporeo/monetario/volumetrico), d. h. die Entsprechungen deutscher N+N-Komposita (Atom-/Körper-/Münz-/Volumengewicht) oder ,peso + Nomen“ (z. B. peso forma; peso gallo/mosca/piuma/welter; peso standard). Vor allem in Gruppe C (mit -gewicht als Letztglied) sind unter den Entsprechungen im Italienischen auch Derivationen und Komposita mit peso (contappeso/soppesare/sovrappeso), wobei die Schreibung (mit $<\mathrm{pp}>$ ) beachtet werden muss. Zuweilen sind die italienischen Bezeichnungen aufgrund ihrer Polysemie etwas verwirrend wie bei Eigengewicht, für das es im zweisprachigen Wörterbuch drei verschiedene Entsprechungen gibt: tara (Verkehrswesen); peso netto (Handel/Wirtschaft) und peso specifico (Naturwissenschaften; Physik). Im Deutschen kann peso netto wiederum sowohl ,Nettogewicht ${ }^{\star}$ als auch ,Füllgewicht' oder, Reingewicht' bedeuten. Für Aufschluss sorgen in vielen Fällen entweder Einträge in Nachschlagewerken oder Bedeutungserklärungen von Fachleuten/Experten.

Differenzen auf lexikalischer und semantischer Ebene gibt es in den Fachgebieten der Wirtschaft, des Verkehrswesens und in der Medizin ${ }^{58}$ i. w. S. Außerdem besteht je nach Verwendungszusammenhang im Vergleich zum Deutschen eine größere terminologische Vielfalt. So unterscheidet das Italienische beim Gewicht (peso) zwischen Masse (carico; massa) und einem schweren Gewicht (carico: ,Last'), wobei die Entsprechungen hier fließend sind.

In bestimmten Fällen besitzen die italienischen Entsprechungen gar keinen Verweis auf peso wie z. B. in Gleichgewicht-equilibrio statt *equipeso. Zuweilen lassen sich auch intersprachliche Unterschiede bei der Verneinung feststellen (wie z. B. in Ungleichgewicht - it. squilibrio) oder in der Bedeutung (3) von Gewicht (kein großes Gewicht haben - non avere molta importanza). Deutsche Wortkombinationen wie ein schweres Gewicht können außerdem nicht wörtlich ins Italienische übersetzt werden (*'un peso pesante').

Die größten Differenzen bestehen bei den Entsprechungen von Redewendungen sowie all jenen Verwendungen, in denen Gewicht übertragen auf ,Bedeutung/Bedeutsamkeit, Wert` referiert

\footnotetext{
${ }^{58} \mathrm{Zu}$ den medizinischen Fachausdrücken gehören in dieser Untersuchung eingedeutschte Bezeichnungen, die auch in der Allgemeinsprache verwendet werden wie z. B. Über- und Untergewicht sowie Ableitungen/Wortbildungen von Gleichgewicht.
} 
und auf ein subjektiv wahrgenommenes, Gewicht'. Viele Bezeichnungen, die im Deutschen einen Bezug zu einem - leichten oder einem schweren - ,Gewicht' haben, werden im Italienischen, je nach Verwendungszusammenhang, nicht ausschließlich mit Bezeichnungen wie peso oder carico wiedergegeben, sondern auch mit Nomina wie z. B. accento/importanza/influenza/portata/prestigio/rilevanza u. a. sowie mit verbalen Wortgruppen (pesare molto; aver(e)/dare importanza) und weiteren Attributen wie z. B. forte/grande bzw. piccolo oder poco, die zwar keinen unmittelbaren Bezug zur Größe Gewicht haben, die aber in Verbindung mit Nomina wie peso den unterschiedlichen Bedeutungen der Gewichtsbezeichnungen im Deutschen entsprechen.

In Tabelle 5 sind intersprachliche lexikalische Aspekte in Bezug auf die polysemen Wörter Gewicht und peso dargestellt. In den beiden Spalten ,BEDEUTUNG' sind die in Abschnitt 2. erläuterten deutschen und italienischen Bedeutungen aufgelistet, d. h. die jeweilige Entsprechung eines deutschen Wortes im Italienischen und umgekehrt (siehe Tabelle 5: Gewicht, das (Sing.) - peso; jeweils in der Bedeutung (1a) / oder in: ,lästig', ,unerträglich` u. a. - peso (in der Bedeutung (6)).

Bezeichnungen, die in den beiden Sprachen in Klammern stehen, sowie leere Zellen bei den Bedeutungen weisen auf eine Null-Entsprechung im Sinne einer fehlenden Gewichtsbezeichnung in der jeweiligen Sprache hin, wie z. B. bei den deutschen Entsprechungen (,anstrengend, unerträglich` u. a) für peso in der Bedeutung (6), da diese deutschen Wörter nicht zur Wortfamilie Gewicht gehören; oder bei italienisch importanza u. a. bzw. corna für Gewicht in den Bedeutungen (3) und (5), die ebenfalls nicht zur Wortfamilie peso zählen. Weitere Leerzellen finden sich z. B. auch bei den italienischen Entsprechungen für wichtig; Wichtigkeit und Gewichtung, da sie ebenfalls keinen lexikalischen Bezug zu peso haben.

\begin{tabular}{|c|c|c|c|}
\hline DEUTSCH & BEDEUTUNG & ITALIENISCH & BEDEUTUNG \\
\hline Gewicht, das (Sing.) & (1a) & peso m (sing.) & (1a) \\
\hline \multirow[t]{2}{*}{ Gewicht } & \multirow[t]{2}{*}{ (1b) } & peso; forza peso & (1b) \\
\hline & & (carico/massa) & \\
\hline Gewichte (Pl.) & (2) & pesi m (pl.) & (2) \\
\hline \multirow[t]{2}{*}{ Gewicht (,Bedeutung') } & \multirow[t]{2}{*}{ (3) } & peso & (3) \\
\hline & & (importanza/rilevanza/accento/enfasi u. а.) & \\
\hline Gewicht (Mathematik) & (4) & peso & (4) \\
\hline $\begin{array}{l}\text { Gewicht ('schweres } \\
\text { Geweih') }\end{array}$ & (5) & $($ corna $($ del capriolo $))$ & \\
\hline $\begin{array}{l}\text { (,Kugel’ ; ,Kugel- } \\
\text { stoßen') }\end{array}$ & & peso (getto (o lancio) del peso) & (2) \\
\hline $\begin{array}{l}\text { (,lästig/unerträglich' } \\
\text { u. a.) }\end{array}$ & & peso & (6) \\
\hline (,Rang/Position') & & peso (Lit.) & (7) \\
\hline \multirow[t]{2}{*}{ gewichten } & \multirow[t]{2}{*}{$(1 \mathrm{~b}) ;(3)$} & soppesare/dare un peso a qc & $(1 b) ;(3)$ \\
\hline & & (valutare qc) & \\
\hline \multirow[t]{2}{*}{ Gewichtung } & $(1 b) ;(2)$ & KE & \\
\hline & (3) & (valutazione) & \\
\hline Gewichtsklasse & $(1 \mathrm{a}) ;(2)$ & categoria di peso & $(1 \mathrm{a}) ;(2)$ \\
\hline Brutto-/Nettogewicht & (1a) & peso lordo/p. netto & (1a) \\
\hline Eigengewicht & (1a) & $($ tara $)$ & \\
\hline
\end{tabular}




\begin{tabular}{|c|c|c|c|}
\hline DEUTSCH & BEDEUTUNG & ITALIENISCH & BEDEUTUNG \\
\hline & (1a) & peso specifico; peso netto & (1a) \\
\hline & (3) & $\mathrm{KE}$ & \\
\hline $\begin{array}{l}\text { Feder-, Fliegen-, } \\
\text { Leicht-, Mittel-, } \\
\text { Schwer-Gewicht }\end{array}$ & (2) & $\begin{array}{l}\text { peso piuma/mosca/leggero, } \\
\text { medio/massimo }\end{array}$ & (2) \\
\hline Gegengewicht & (2); (3) & contrappeso & (2); (3) \\
\hline Gleichgewicht & $\begin{array}{l}(1 \mathrm{a}) ; \mathrm{b}) ;(2) ; \\
\text { fig. }\end{array}$ & (equilibrio) & \\
\hline \multirow[t]{2}{*}{ Körpergewicht } & \multirow[t]{2}{*}{ (1a) } & peso corporeo & (1a) \\
\hline & & (massa) & \\
\hline \multirow[t]{2}{*}{ Leergewicht } & \multirow[t]{2}{*}{ (1a) } & peso a vuoto & (1a) \\
\hline & & peso morto ${ }^{59}$ & (1a); fig. \\
\hline \multirow[t]{2}{*}{ Übergewicht } & (1a) & sovrappeso & (1a) \\
\hline & (3) & (predominio, prevalere su) & \\
\hline \multirow[t]{3}{*}{ gewichtig } & (1a) & (grosso); (corpacciuto) & \\
\hline & \multirow[t]{2}{*}{ (3) } & di un certo peso & (3) \\
\hline & & (importante/autorevole/di prestigio) & \\
\hline wichtig & (3) & (importante) & \\
\hline Wichtigkeit & (3) & (importanza) & \\
\hline Wichte & (1a) & peso specifico & (1a) \\
\hline Wucht $^{60}$ & fig. & (forza/violenza/impeto; cannonata/schianto $)$ & \\
\hline
\end{tabular}

Tabelle 5: Deutsche und italienische Gewichtsbezeichnungen

Bei den Gewichtsbezeichnungen und Messgrößen (Handel; Sport) sowie bei Bezeichnungen in den Bedeutungen (1a) und (2) (siehe 2.1) herrschen die größten Übereinstimmungen. Fehlende Einträge bzw. Null-Entsprechungen von Gewichtsbezeichnungen in allgemeinsprachlichen zweisprachigen Wörterbüchern konnten teilweise - sowohl im Italienischen als auch im Deutschen - in einsprachigen Wörterbüchern und Nachschlagewerken gefunden werden.

In der Bedeutung (3) kann das deutsche Wort Gewicht im Italienischen neben peso auch mit einer Reihe Nomina ohne Bezug zu peso, oftmals in Verbindung mit adjektivischen Attributen, wiedergegeben werden (siehe 3.1.1; Tabelle 3; 3.3.2).

\section{$5 \quad$ Fazit und Ausblick}

Im Zusammenhang mit der Basisgröße ,Gewicht' gibt es im Deutschen und im Italienischen eine Vielzahl von Bezeichnungen, die den unterschiedlichen Bedeutungen dieser Messgröße entsprechen. Ein kontrastiver Vergleich aus lexikalischer Perspektive konnte in Bezug auf die Wortformen und die Bedeutungen sowohl Übereinstimmungen als auch Abweichungen in beiden Sprachen feststellen.

Während man im Deutschen allgemeinsprachlich nicht zwischen Gewicht und Gewichtskraft unterscheidet, macht man im Italienischen einen Unterschied, ob die , gewisse Schwere eines Körpers` gemeint ist, also die Masse eines Körpers (it. massa), oder ob es sich um

\footnotetext{
59 In einer bildhaften Bedeutung steht diese Bezeichnung (,peso morto“) für ,Ballast; unnötige Last; Klotz am Bein“ (cf. Giacoma/Kolb 2019: 2100).

${ }^{60} \mathrm{Im}$ einsprachigen Wörterbuch bedeutet „Wucht“: ,durch Gewicht, Kraft, Schwung o. Ä. erzeugte Heftigkeit, mit der sich ein Körper gegen jemanden, etwas bewegt, auf jemanden, etwas auftrifft‘ (cf. Duden online s. v. Wucht $)$.
} 
Gewichtskraft (it. peso/forza peso) handelt. Eine Übereinstimmung im Sinne von „Gewicht gleich peso" besteht vor allem bei den Gewichten (it. pesi), die beim Wiegen eingesetzt werden, beim Körpergewicht und im Sport. In Verwendungszusammenhängen, in denen Gewicht eine bildhafte Bedeutung im Sinne einer subjektiv wahrgenommenen Größe hat, variieren die sogenannten Gewichtsbezeichnungen in beiden Sprachen. Auch in der Gegenüberstellung deutscher und italienischer Redewendungen mit der Komponente Gewicht bzw. mit peso wurden Übereinstimmungen im Sinne „Gewicht gleich peso“ und „peso gleich Gewicht“ festgestellt. Es gibt jedoch auch eine Reihe von Redewendungen, in denen in der einen Sprache Gewichtsbezeichnungen und in der anderen Sprache andere Komponenten verwendet werden. Untersuchungen über die Wortfamilie oder das spezielle Wortfeld ,Gewicht' aus deutscher und italienischer Perspektive unter Einbezug weiterer Wortklassen und Kontexte werden größeren Aufschluss über die komplexen Zusammenhänge - Gemeinsamkeiten und Unterschiede - im deutsch-italienischen Sprachvergleich geben.

\section{Literaturverzeichnis}

Arbeitsgemeinschaft der öffentlich-rechtlichen Rundfunkanstalten der Bundesrepublik Deutschland (ARD): ard.de [04.12.2020].

Bocaccio, Giovanni (1866): Il Decamerone. Con spiegazioni tratte dai migliori commentari. Volume Secondo. Moutier: Paris.

Bocaccio, Giovanni (1783): Der Decameron des Boccaz. Aus dem Italiänischen neu übersezt. Zweiter Band. St. Petersburg.

Brockhaus Enzyklopädie online: brockhaus.de/info/ [04.12.2020].

Deutsches Rechtswörterbuch (DRW). Forschungsstelle der Heidelberger Akademie der Wissenschaften. drw-www.adw.uni-heidelberg.de/drw-cgi/zeige [10.10.2020]

Deutsches Referenzkorpus DeReKo, IDS, Mannheim: cosmas2.ids-mannheim.de/cosmas2web/ [04.10.2021].

Deutschlandradio, Programm: Deutschlandfunk(Dlf): deutschlandfunk.de [14.12.2020].

Deutschlandradio, Programm: Deutschlandfunk Kultur (Dlf Kultur): deutschlandfunkkultur.de [14.12.2020].

Devoto, Giacomo et al. (2017): Nuovo Devoto-Oli. Il Vocabolario dell'italiano contemporaneo. Milano: Le Monnier.

Dienstblatt der Hochschulen des Saarlandes (2005): „Prüfungsordnung für den Bachelorstudiengang Mathematik und die Masterstudiengänge Angewandte Mathematik“ 13: 144, §13, (11): 171, Saarbrücken, 17.05.2005. math.uni-sb.de/pdf/PO_B.pdf [04.10.2021].

Digitales Wörterbuch der deutschen Sprache (DWDS): dwds.de [04.10.2021].

Duden (2020a): Band 11: Redewendungen. Wörterbuch der deutschen Idiomatik. 5. vollständig überarbeitete und erweiterte Auflage. Berlin: Dudenverlag.

Duden (2020b): Band 7: Das Herkunftswörterbuch: Etymologie der deutschen Sprache. 6. vollständig überarbeitete und erweiterte Auflage. Berlin: Dudenverlag.

Duden (2019): Deutsches Universalwörterbuch. 9., überarbeitete und erweiterte Auflage. Berlin: Dudenverlag.

Duden (2018): Band 10. Das Bedeutungswörterbuch. 5. Aufl. Berlin: Dudenverlag.

Duden (2014): Wer hat den Teufel an die Wand gemalt. Redensarten - wo sie herkommen, was sie bedeuten. Berlin: Dudenverlag. 
Duden Wörterbuch online: Duden. de/woerterbuch [04.10.2021].

Fleischer, Wolfgang/Barz, Irmhild (1995): Wortbildung der deutschen Gegenwartssprache. 2. Aufl. Tübingen: Niemeyer.

Gabler Wirtschaftslexikon: wirtschaftslexikon.gabler.de/ [20.09.2020].

Giacoma, Luisa/Kolb, Susanne (2019) (eds.): Il nuovo dizionario di tedesco. Dizionario Tedesco-Italiano; Italiano-Tedesco. 4. Aufl. Bologna/Stuttgart: Zanichelli Pons.

Giacoma, Luisa/Kolb, Susanne (2016) (eds.): Il tedesco smart, Dizionario Tedesco-Italiano; Italiano-Tedesco. 2. Aufl. Bologna, Stuttgart: Zanichelli Pons.

Giordano, Guglielmo (1999): Tecnica delle costruzioni in legno. $5^{\text {a }}$ edizione aggiornata con la collaborazione di Ario Ceccotti Luca Uzielli. Milano: Hoepli.

Goethe, Johann Wolfgang von (1982/div. Erstdr. ab 1773): Schriften zur Kunst. Schriften zur Literatur. Maximen und Reflexionen. Goethes Werke, Bd. 12. München: Beck.

Hamburger Renn-Club e.V., Galopprennbahn Hamburg-Horn: galopp-hamburg.de [21.10.2021].

Hammerschmid-Gollwitzer, Josef (1981): Wörterbuch der medizinischen Fachausdrücke. München: RVG.

Haustein, Heinz-Dieter (2001): Weltchronik des Messens. Universalgeschichte von Maß, Zahl und Gewicht. Berlin/New York: de Gruyter.

Horn, Christoph/Rapp, Christof (eds.) (2008): Wörterbuch der antiken Philosophie. 2. überarbeitete Auflage. München: Beck.

Kluge, Friedrich/Götze, Alfred (2011): Etymologische Wörterbuch der deutschen Sprache. Berlin/New York: de Gruyter.

Koch, Ildikó (2000): Die Metataxe im deutsch-italienischen Sprachvergleich. Eine Studie der verbbedingten Abweichungen im Satzbau. Frankfurt am Main: Lang.

Lenz, Harald Othmar (1873): Gemeinnützige Naturgeschichte: Die Säugethiere. Band 1. Gotha: Thienemann's Hofbuchhandlung.

Mackensen, Lutz (1981): 10000 Zitate, Redensarten, Sprichwörter nach Anfängen und Stichwörtern alphabetisch geordnet. Wiesbaden: VMA-Verlag.

Nocentini, Alberto di, con la collaborazione Alessandro Parenti (2010): L'etimologico. Vocabolario della lingua italiana. Milano: Le Monnier.

Offizielles Stadtportal für Hamburg, Hafen-ABC: hamburg.de/hafen-abc/\#anker_3 [04.09.2021].

Pera, Franz/Schmiedebach, Heinz-Peter (2010): Medizinischer Wortschatz. Terminologie kompakt. 2. Aufl. Berlin/New York: de Gruyter.

Preuß, Hugo (2009): Gesammelte Schriften. Zweiter Band. Öffentliches Recht und Rechtsphilosophie im Kaiserreich. Tübingen: Mohr Siebeck.

Rocco, Goranka (2020): „Morphosyntaktische, semantisch-lexikalische und funktionale Aspekte der Numeruskategorie im Deutsch-Italienisch-Vergleich und ihre sprach- und übersetzungsdidaktischen Implikationen“. German as a foreign language (GfFL) 2/2020: 1-22. arts.units.it/retrieve/handle/11368/2973622/341928/Rocco_Morphosyntaktische\%20semantisch-lexikalische\%20und.pdf [04.12.2020].

Römer, Christine (2010): „,Wortfamilie, die“,Wortfeld, das““. In: Barkowski, Hans/Krumm, Hans-Jürgen (eds.): Fachlexikon Deutsch als Fremd- und Zweitsprache. Tübingen/Basel, Francke: 359. 
Schemann, Hans/Birkenhauer, Renate (1992): Synomymwörterbuch der deutschen Redensarten. Stuttgart/Dresden: Klett.

Selig, Maria/Morlicchio, Elda/Dittmar, Norbert (eds.) (2016): Gesprächsanalyse zwischen Syntax und Pragmatik. Deutsche und italienische Konstruktionen. Tübingen: Stauffenburg.

Sieveking, Robert (2019): Die Geschäftsbedingungen des Waren-Vereins der Hamburger Börse e. V.: Auf Grund der Rechtsprechung seines Schiedsgerichts und der Handelsbräuche seines Bereichs, edited by Otto Mathies and Walter Grimm. Berlin/Boston: de Gruyter.

Treccani Vocabolario online: treccani.it [20.10.2021].

Troike Strambaci, Hannelore/Helffrich Mariani, Elisabeth (2009): Wörterbuch für Recht und Wirtschaft/Vocabolario del diritto e del'economia. Band I: Deutsch - Italienisch/Tomo I: Tedesco-Italiano. 3. Auflage, München/Mailand: Beck.

Reverso Übersetzungsportal: context.reverso.net/übersetzung/ [04.09.2018].

Wanzeck, Christiane (2010): Lexikologie. Göttingen: Vandenhoeck \& Ruprecht.

Zweites Deutsches Fernsehen (ZDF): zdf.de [04.12.2020]. 